\title{
Comparisons within the Rice GA 2-Oxidase Gene Family Revealed Three Dominant Paralogs and a Functional Attenuated Gene that Led to the Identification of Four Amino Acid Variants Associated with GA Deactivation Capability
}

Kun-Ting Hsieh ${ }^{1}$, Yi-Ting Chen ${ }^{1}$, Ting-Jen Hu', Shih-Min Lin ${ }^{1}$, Chih-Hung Hsieh ${ }^{1}$, Su-Hui Liu', Shiau-Yu Shiue ${ }^{1}$, Shuen-Fang Lo' ${ }^{2}$ I-Wen Wang ${ }^{3}$, Ching-Shan Tseng ${ }^{3}$ and Liang-Jwu Chen ${ }^{1,2^{*}}$ (i)

\begin{abstract}
Background: GA 2-oxidases (GA2oxs) are involved in regulating GA homeostasis in plants by inactivating bioactive GAs through $2 \beta$-hydroxylation. Rice GA2oxs are encoded by a family of 10 genes; some of them have been characterized, but no comprehensive comparisons for all these genes have been conducted.

Results: Rice plants with nine functional GA2oxs were demonstrated in the present study, and these genes not only were differentially expressed but also revealed various capabilities for GA deactivation based on their heightreducing effects in transgenic plants. Compared to that of wild-type plants, the relative plant height (RPH) of transgenic plants was scored to estimate their reducing effects, and 8.3\% to 59.5\% RPH was observed. Phylogenetic analysis of class I GA20x genes revealed two functionally distinct clades in the Poaceae. The OsGA2ox3, 4, and 8 genes belonging to clade A showed the most severe effect ( $8.3 \%$ to $8.7 \% \mathrm{RPH})$ on plant height reduction, whereas the OsGA20x7 gene belonging to clade B showed the least severe effect (59.5\% RPH). The clade A OsGA2ox3 gene contained two conserved C186/C194 amino acids that were crucial for enzymatic activity. In the present study, these amino acids were replaced with OsGA20X7-conserved arginine (C186R) and proline (C194P), respectively, or simultaneously (C186R/C194P) to demonstrate their importance in planta. Another two amino acids, Q220 and Y274, conserved in OsGA20x3 were substituted with glutamic acid (E) and phenylalanine (F), respectively, or simultaneously to show their significance in planta. In addition, through sequence divergence, RNA expression profile and GA deactivation capability analyses, we proposed that OsGA20x1, OsGA20x3 and OsGA20x6 function as the predominant paralogs in each of their respective classes.
\end{abstract}

\footnotetext{
* Correspondence: ljchen@nchu.edu.tw

'Institute of Molecular Biology, National Chung Hsing University, Taichung

40227, Taiwan

2Biotechnology Center, National Chung Hsing University, Taichung 40227,

Taiwan

Full list of author information is available at the end of the article
}

(อ) The Author(s). 2021 Open Access This article is licensed under a Creative Commons Attribution 4.0 International License, which permits use, sharing, adaptation, distribution and reproduction in any medium or format, as long as you give appropriate credit to the original author(s) and the source, provide a link to the Creative Commons licence, and indicate if changes were made. The images or other third party material in this article are included in the article's Creative Commons licence, unless indicated otherwise in a credit line to the material. If material is not included in the article's Creative Commons licence and your intended use is not permitted by statutory regulation or exceeds the permitted use, you will need to obtain permission directly from the copyright holder. To view a copy of this licence, visit http://creativecommons.org/licenses/by/4.0/. 
Conclusions: This study demonstrates rice has nine functional GA20xs and the class I GA20x genes are divided into two functionally distinct clades. Among them, the OsGA20X7 of clade $B$ is a functional attenuated gene and the OsGA20x1, OsGA2OX3 and OsGA20x6 are the three predominant paralogs in the family.

Keywords: Duplicate genes, Expression profile, GA 2-oxidase gene family, GA deactivation capability, Phylogenetic footprinting, Regulatory hypofunctionalization, Sequence divergence

\section{Background}

The phytohormone gibberellin (GA) regulates a broad spectrum of plant growth and development aspects, including seed development, germination, stem elongation, leaf expansion, flowering and fruit development (Yamaguchi 2008). Although more than 130 GAs have been identified (MacMillan 2001), only $\mathrm{GA}_{1}, \mathrm{GA}_{3}, \mathrm{GA}_{4}$ and $\mathrm{GA}_{7}$ are recognized as major bioactive GAs in plants. The availability of bioactive GAs in plant tissues must be homeostatically maintained for proper plant growth, which is precisely regulated by both GA biosynthetic and catabolic enzymes.

The late stage of GA biosynthesis and GA catabolism in the cytosol are governed by three types of GA oxidases in the 2-oxoglutarate-dependent dioxygenase (2OGD) enzyme family (Kawai et al. 2014). The GA 20oxidase (GA20ox), GA 3-oxidase (GA3ox) and GA 2oxidase (GA2ox) members of the 2OGD family are thought to be the main enzymes involved in regulating GA homeostasis in plants during growth and in response to environmental signals (Yamaguchi 2008). Among them, GA20oxs and GA3oxs are GA biosynthetic enzymes (Toyomasu et al. 1997; Itoh et al. 2001), and GA2oxs are recognized as GA deactivation enzymes that convert GA precursors or bioactive GAs into inactive GAs by 2 $\beta$-hydroxylation (Thomas et al. 1999). GA2ox is prevalent in seed plant species and encoded by gene family: for instance, nine in Arabidopsis (Lange and Lange 2020), ten in rice (Lo et al. 2008), eleven in Brachypodium distachyon (Pearce et al. 2015), eight in tomato (Chen et al. 2016) and 14 in Medicago truncatula (Kim et al. 2019) have been previously reported. Phylogenetic analysis based on protein sequences classified GA2oxs into three classes (Additional file: Fig. S1, Lee and Zeevaart 2005): class I and class II, whose members catalyze $\mathrm{C}_{19}$-GAs, are referred to as $\mathrm{C}_{19}$-type GA2oxs, and class III, whose members catalyze $\mathrm{C}_{20}$-GAs, are referred to as $\mathrm{C}_{20}$-type GA2oxs (Schomburg et al. 2003; Lo et al. 2008).

To explore how GA2ox can form a large gene family and how each gene evolved, numerous evolutionary analyses have been performed in land plant lineages (Han and Zhu 2011; Kawai et al. 2014; Huang et al. 2015; Takehara et al. 2020; Yoshida et al. 2020). Phylogenetic analysis showed that the gene number of GA2oxs expanded and evolved independently after the divergence of eudicots and monocots (Han and Zhu 2011; Kawai et al. 2014; Takehara et al. 2020; Yoshida et al. 2020). The rapid expansion of GA2oxs in eudicots and monocots was thought to be due to large-scale genome duplications (Huang et al. 2015); the duplicated genes may have either lost their function or retained their function, but their promoter and/or coding sequences diverged over time to form genes with various expression patterns and functions in the family (Panchy et al. 2016).

The duplicated genes that lost their function could have been the result of nonfunctional mutations in coding regions that resulted in premature stop codons and/ or shifts in reading frame or in intro-exon structure (Balakirev and Ayala 2003), or the loss of function also could have been the result of sequence mutations within the promoter region that deactivated the expression, which could also lead to loss of its function; both of these are processes of gene pseudogenization (Yang et al. 2011). In addition, genome-wide comprehensive analysis of pseudogenes in rice and Arabidopsis showed that the coding regions of these pseudogenes tend to present increased amounts of nonsynonymous substitutions (Zou et al. 2009). Other than encountering pseudogenization, the duplicated genes in the family would also encounter sequence divergence in the regulatory and/or coding regions that caused RNA expression and protein function divergence, causing these genes to undergo either regulatory hypofunctionalization or subfunctionalization (Duarte et al. 2006; Panchy et al. 2016). Regulatory hypofunctionalization is where one member of a paralogous pair has an overall decrease in expression level while maintaining its protein function (Duarte et al. 2006). The expression difference between duplicated genes is a common phenomenon in plants, as $70 \%$ of duplicated gene pairs from Arabidopsis have different expression profiles (Ganko et al. 2007), and this phenomenon has also been observed in rice ( $\mathrm{Li}$ et al. 2009) and cotton (Renny-Byfield et al. 2014). This divergence in expression among duplicated genes is negatively correlated with nonsynonymous substitution rates in the coding region ( $\mathrm{Li}$ et al. 2005; Ganko et al. 2007), meaning that divergence in expression tends to evolve at the time when protein function becomes conserved in a family. The presence of duplicated genes retaining functional redundancy but with reduced expression could represent a way to maintain duplicates in a family (Qian 
et al. 2010). Additionally, duplicated genes could function as genetic buffers to help plants overcome various environmental stresses (Duarte et al. 2006; Zhang 2012; Illouz-Eliaz et al. 2019).

Like all other duplicated genes in a family, each GA2ox gene is differentially expressed in various tissues at different growth stages (Rieu et al. 2008; Giacomelli et al. 2013; Chen et al. 2016; Li et al. 2019), indicating that the divergence in expression of each individual GA2ox gene corresponds to different evolved biological functions. For example, SlGA2ox7, which is highly expressed in the stems (Chen et al. 2016), was suggested to be responsible for the regulation of stem-specific elongation in tomato (Schrager-Lavelle et al. 2019); $M t G A 2 o x 10$, which is expressed in symbiotic tissues and nodules, was proposed to be involved in the regulation of rhizobial infection and nodule development in $M$. truncatula (Kim et al. 2019); and in rice, OsGA2ox1 expressed around the shoot apex was proposed to be involved in the regulation of phase transition (Sakamoto et al. 2001), whereas OsGA2ox3 was homeostatically regulated by bioactive GAs, which was suggested to play an important role in GA homeostasis (Sakai et al. 2003). Moreover, the expression of OsGA2ox4 is upregulated by light and affects internode elongation, which might contribute to lodging resistance (Hirose et al. 2012; Liu et al. 2018). These expressional and functional correlations could have been evolved from sequence divergences between duplicated copies in those families ( $\mathrm{Li}$ et al. 2005; Moghe and Last 2015).

Indeed, differential RNA expression resulting from sequence differences in the regulatory regions in many hormone-responsive genes and GA biosynthetic and catabolic genes has been observed. For example, within the Arabidopsis GA2ox gene family, the AtGA2ox6 promoter contains the AGL15 binding motif (CCAATTTA ATGG) and the absence of AGL15 reduces AtGA2ox6 expression (Wang et al. 2004), whereas the AtGA2ox7 promoter contains a DRE-like motif (GCCGAC and ATCGAC), and the expression of this gene is strongly upregulated during high-salinity stress (Magome et al. 2008). In addition, the expression of AtGA2ox2 and $A t G A 2 o x 4$ is upregulated by KN1-like homeobox (KNOX) proteins to reduce bioactive GA levels in the shoot apical meristem (SAM) (Jasinski et al. 2005). Similarly, in maize, ZmGA2ox1 contains a KNOTTED1 (KN1) binding site in the first intron that is regulated by KNOX and is responsible for the bioactive GA levels around the SAM (Bolduc and Hake 2009). In sorghum, several cis-regulatory elements related to $\mathrm{ABA}$ and $\mathrm{GA}$ signaling were identified in the $S b G A 2 o x 3$ promoter region responsible for different degrees of dormancy between dormant IS9530 and less dormant Redland B2 grains (Rodriguez et al. 2012; Cantoro et al. 2013). The
SD1 ${ }^{\mathrm{C} 9285}$ (OsGA20ox2 in C9285) haplotype includes 17 specific polymorphisms in the promoter and second intron region that affect the differential expression of OsGA20ox2 between C9285 and Taichung 65 under submergence or ethylene treatment (Kuroha et al. 2018). Moreover, a comprehensive report showed that sequence-specific response elements in the regulatory regions are highly conserved in many hormoneresponsive genes among angiosperms to regulate their hormone responses, and any replacement of the conserved sequences affected their tissue-specific expression (Lieberman-Lazarovich et al. 2019).

The functional divergence among genes in a family might also have evolved from amino acid sequence variations (Moghe and Last 2015; Panchy et al. 2016). Several studies have shown that amino acid sequence differences in rice GA2ox and GA20ox significantly influence their protein activities. Our previous study demonstrated that different amino acid replacements in OsGA2ox6 affected GA deactivation activities in plants to varying degrees (Lo et al. 2017), and a single sequence variation in the Shortened Basal Internodes (SBI, OsGA2ox4) allele that caused an amino acid substitution at position G338R of OsGA2ox4 significantly affected its activity (Liu et al. 2018). The crystal structure of OsGA2ox3 shows that the amino acid C194 is crucial for tetramer formation and is responsible for the approximately 9-fold $K m$ and 4-fold $V$ max values of the monomer, and this $\mathrm{C} 194$ amino acid was conserved only in OsGA2ox3, 4 and 8 but diverged in other members in the family (Takehara et al. 2020). Another study showed that an OsGA20ox2 allele in the deep-water rice variety C9285 has two amino acid substitutions that lead to a 271-fold increase in activity compared with that in Taichung 65 , and this substitution, which is functionally distinct, naturally evolved and was then selected by breeders for deep-water rice cultivation (Kuroha et al. 2018).

In rice, although the biological roles of OsGA2ox1, 3, and 4 corresponding to their specific expression patterns have been characterized (Sakamoto et al. 2001; Sakai et al. 2003; Liu et al. 2018), no comprehensive comparison of expression profiles for all OsGA2oxs has been undertaken. Moreover, since the substrate preference of GA2ox proteins could be different among the members of the family (Lange and Lange 2020) and their GA deactivation capability could also vary by amino acid sequence divergence (Lo et al. 2017; Liu et al. 2018; Takehara et al. 2020), to evaluate the functional correlation and importance for each OsGA2ox gene in the family, apart from the expression profiles, additional determinants such as their sequence differences in the regulatory and coding regions as well as their GA deactivation capability should be included. 
Gene functional studies rely mostly on loss-offunction knockout mutants; however, knockout mutants of one member of the family usually show no discernible phenotype due to the functional redundancy existing among other members of that family (Rutter et al. 2017). Therefore, for a family of 10 genes, such as the rice GA2ox family, exploring the function of each individual gene through a knockout mutant approach is not always feasible. Thus far, only a limited number of single GA2ox knockout mutants in tomato $C_{20}$-type SlGA2ox7 (Schrager-Lavelle et al. 2019), M. truncatula $C_{20}$-type $M t G A 20 x 10$ (Kim et al. 2019) and rice OsGA2ox3 (Takehara et al. 2020) in a large family with discernible phenotypes have been reported. In contrast, T-DNA activation-tagged mutants that lead to activation of a target gene that causes obvious phenotypic changes can facilitate the functional study of OsGA2ox genes (Lo et al. 2008). In addition to T-DNA activation-tagged mutants, transgenic plants ectopically overexpressing OsGA2ox genes could cause a quantifiable reduced-plant height phenotype and have been used to study their functional effects in planta (Lo et al. 2017). This overexpression approach that causes a quantifiable dwarf phenotype not only can be used to identify the functional effects of each gene but also can be used to compare all their GA deactivation capabilities in planta by quantifying the degree to which they reduce plant height.

In the present study, other than four previously characterized (OsGA2ox3, 5, 6 and 9 genes) mutants (Lo et al. 2008), five additional (OsGA2ox1, 2, 4, 7 and 8 genes) uncharacterized T-DNA activation-tagged mutants were analyzed and used to facilitate the cloning of the full-length cDNA of their respective genes. In addition, transgenic plants overexpressing the full-length cDNA of each of the OsGA2ox genes ectopically in the same rice variety were compared to investigate their effects on plant height reduction, and their reducing effects were scored on the basis of relative plant height $(\mathrm{RPH})$ to estimate their GA deactivation capability in planta. The importance and significance of four amino acids conserved in clade A but not in clade B of class I GA2oxs, which are crucial for GA deactivation, have been demonstrated by transgenic approaches in planta. In addition, through large-scale sequence comparisons of rice GA2ox genes from 4276 rice accessions (Zhao et al. 2015) and the GA2ox expression profiles collected from available databases (UniVIO: http://univio.psc. riken.jp/; RED IC4R: http://ic4r.org; RiceXPro; https:// ricexpro.dna.affrc.go.jp/), as well as analysis of the GA deactivation capability of transgenic plants, three dominant paralogs in the family in each of their respective classes were revealed.

\section{Results}

Functional Screening of Rice GA2ox1, GA2ox2, GA2ox4, GA20x7 and GA20x8 Using T-DNA Activation-Tagged Mutants

Our previous study showed that enhanced expression of OsGA2ox3 (M77777), OsGA2ox5 (M27337), OsGA2ox6 (M47191) and OsGA2ox9 (M58817) in their respective T-DNA activation-tagged mutants reduced plant height, which provided a useful tool for functional screening of GA2ox genes (Lo et al. 2008). To complete the functional screening of all GA2ox genes in the family, five additional T-DNA activationtagged mutants, M36548 (for OsGA2ox1), M43852 (for OsGA2ox2), M96803 (for OsGA2ox4), M66925 (for OsGA2ox7) and M61685 (for OsGA2ox8), were selected from the Taiwan Rice Insertional Mutant (TRIM) library (Hsing et al. 2007) through a reverse genetic approach.

Among them, the T-DNA in mutant M36548 was inserted $5.9 \mathrm{~kb}$ upstream from OsGA2ox1 (Fig. 1a), and the T-DNA in mutant M43852 was inserted approximately $12.5 \mathrm{~kb}$ upstream from a putative OsGA2ox2 (LOC_Os01g22920) (Fig. 1b). In mutants M96803 (OsGA2ox4), M66925 (OsGA2ox7) and M61685 (OsGA2ox8), their T-DNAs were inserted either $2 \mathrm{~kb}$ upstream, $19 \mathrm{~kb}$ upstream or $15.5 \mathrm{~kb}$ downstream from their respective target OsGA2ox genes (Fig. 1c-e). Varying degrees of plant height reduction ranging from approximately 30\% (70\% RPH for mutants M43852, M96803 and M61685) to 4\% (96\% RPH for mutants M36548 and M66925) in their respective homozygous mutant lines were observed (Fig. 1f), suggesting that the genes were all functional. The difference in plant heightreducing effects among the mutants might be caused by various GA deactivation capabilities and/or different degrees of target gene activation among them.

To support the phenomenon that plant heightreducing effects in mutants are correlated with the activation of their target genes, the RNA expression of each OsGA2ox target gene in the T1 progeny, including segregated wild-type (W/W), heterozygous (T/ $\mathrm{W})$ and homozygous $(\mathrm{T} / \mathrm{T})$ plants of their respective mutants, was analyzed (Fig. 1a-e, top row of each gel). Except for the putative OsGA2ox2 gene (LOC_ Os01g22920), the results showed that the expression of each target gene in their respective mutants was activated and correlated with their reduced-plant height phenotypes. Although expression of the LOC_ Os01g22920 gene was not detected, reduced height of its respective mutant M43852 was observed (Fig. 1b), suggesting that the annotation of LOC_Os01g22920 for $O s G A 20 \times 2$ was not correct or that the reducedplant height phenotype might be caused by other unidentified T-DNA insertion events. 

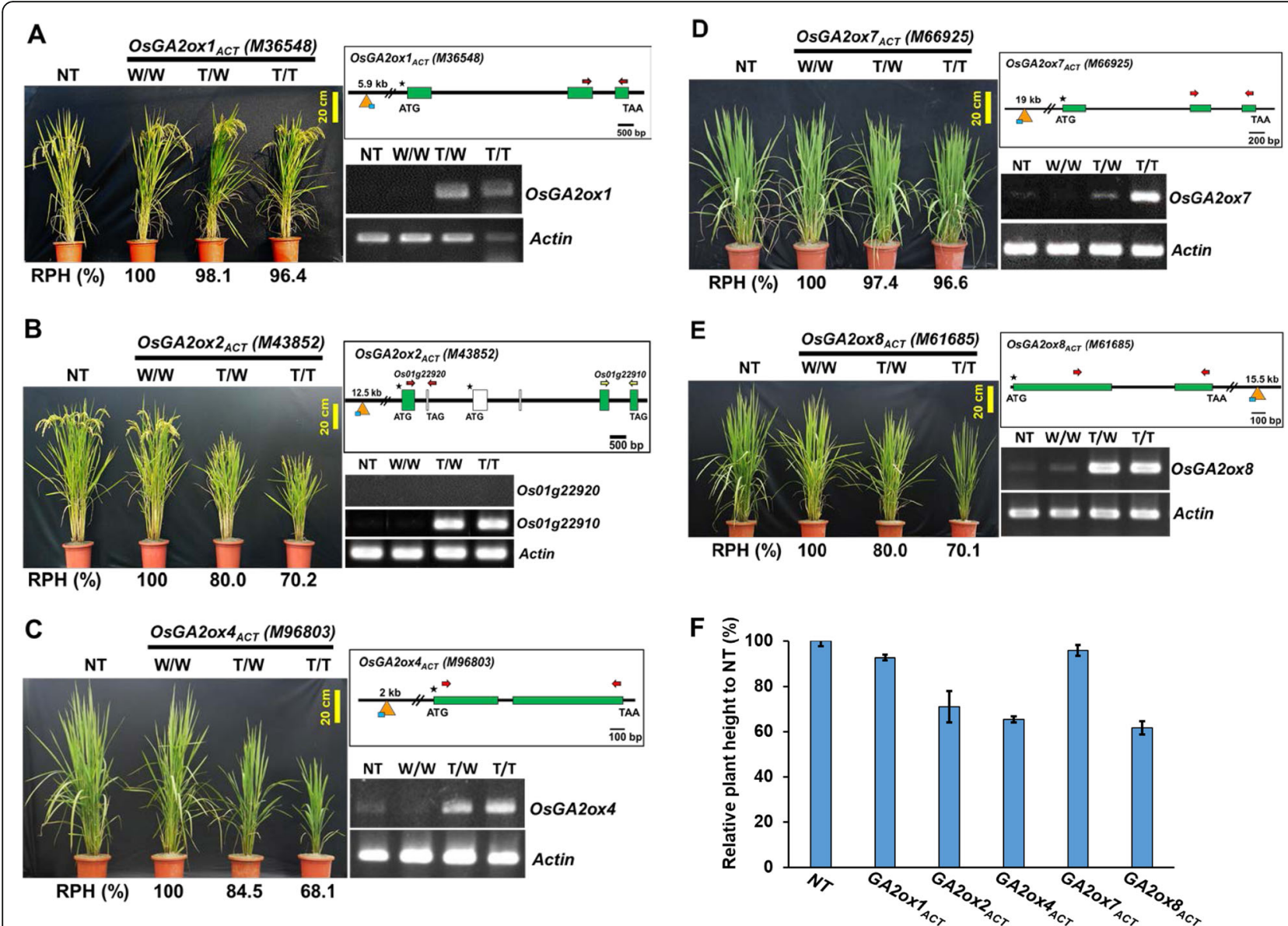

Fig. 1 Phenotypes and expression characterization of the T-DNA activation-tagged mutants for OSGA2OX1, 2, 4, 7, and 8. The phenotypes and relative plant height (RPH \%) of T-DNA activation-tagged mutants (with various genotypes (W/W, segregated WT; T/W, heterozygous; T/T, homozygous)) compared with those of nontransgenic plants (NT) and the RT-PCR analysis results of target gene activation are shown. The Actin gene was used as a control. The T-DNA insertion sites (orange triangles) relative to the ATG start codon of their target genes are shown in the graphic adjacent to the images. a The T-DNA mutant M36548 (an OsGA20x1 ACT mutant), with the T-DNA inserted 5.9 kb upstream from OsGA20x1, is shown. $\mathbf{b}$ The T-DNA mutant M43852 (an OsGA20x2 ACT mutant), with the T-DNA inserted $12.5 \mathrm{~kb}$ upstream from OsGA20x2, is shown. c The TDNA mutant M96803 (an OsGA20x4 ACT mutant), with the T-DNA inserted 2 kb upstream from OsGA20x4, is shown. d The T-DNA mutant M66925

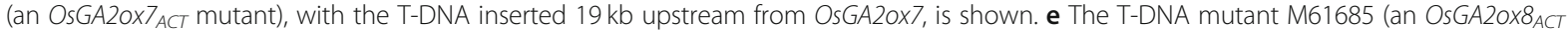
mutant), with the T-DNA inserted $15.5 \mathrm{~kb}$ downstream from OsGA20x8, is shown. $\mathbf{f}$ RPH comparison of the homozygous plants of the above TDNA activation-tagged mutants. Values are means \pm SE $(n=10)$

\section{The OsGA2ox2 Gene Contains a Long First Intron and Three Exons that Encode 370 Amino Acids, with Two Typical Conserved Protein Domains}

To confirm that the mutant M43852 contained no extra T-DNA insertions, a Southern blotting assay using XhoIdigested genomic DNA hybridized by a ${ }^{32}$ P-labeled GUS DNA probe was performed (Fig. 2a). The results showed that mutant M43852 contained only one copy of TDNA inserted $12.5 \mathrm{~kb}$ upstream from LOC_Os01g22920, which was revealed by the expected $8.3 \mathrm{~kb}$ hybridized signal (Fig. 2a). The gene structure of the putative OsGA2ox2-LOC_Os01g22920 and the flanking genes 20 $\mathrm{kb}$ up- and downstream from the T-DNA insertion site were then checked, and five genes, named gene-01 to gene-05, including the putative OsGA2ox2 (LOC_
Os01g22920 is gene-03), were annotated in this $40 \mathrm{~kb}$ region (Fig. 2b). A series of schematic diagrams are provided to show the relative locations of these annotated genes, their transcription directions and the location of the T-DNA insertions (Fig. 2b). Also shown is an enlarged region containing the exons/introns, the start codon (ATG) and stop codon (TAG) of annotated gene03 and gene-04 (LOC_Os01g22910) (Fig. 2c), and their proposed spliced cDNA with the expected size of RTPCR products (Fig. 2d) using the primer sets indicated with colored arrows (Fig. 2c).

Among these flanking genes, LOC_Os01g22910 was located downstream of LOC_Os01g22920 and was strongly activated in mutant M43852 (Figs. 1b; 2e), and this gene activation was associated with a reduction in 

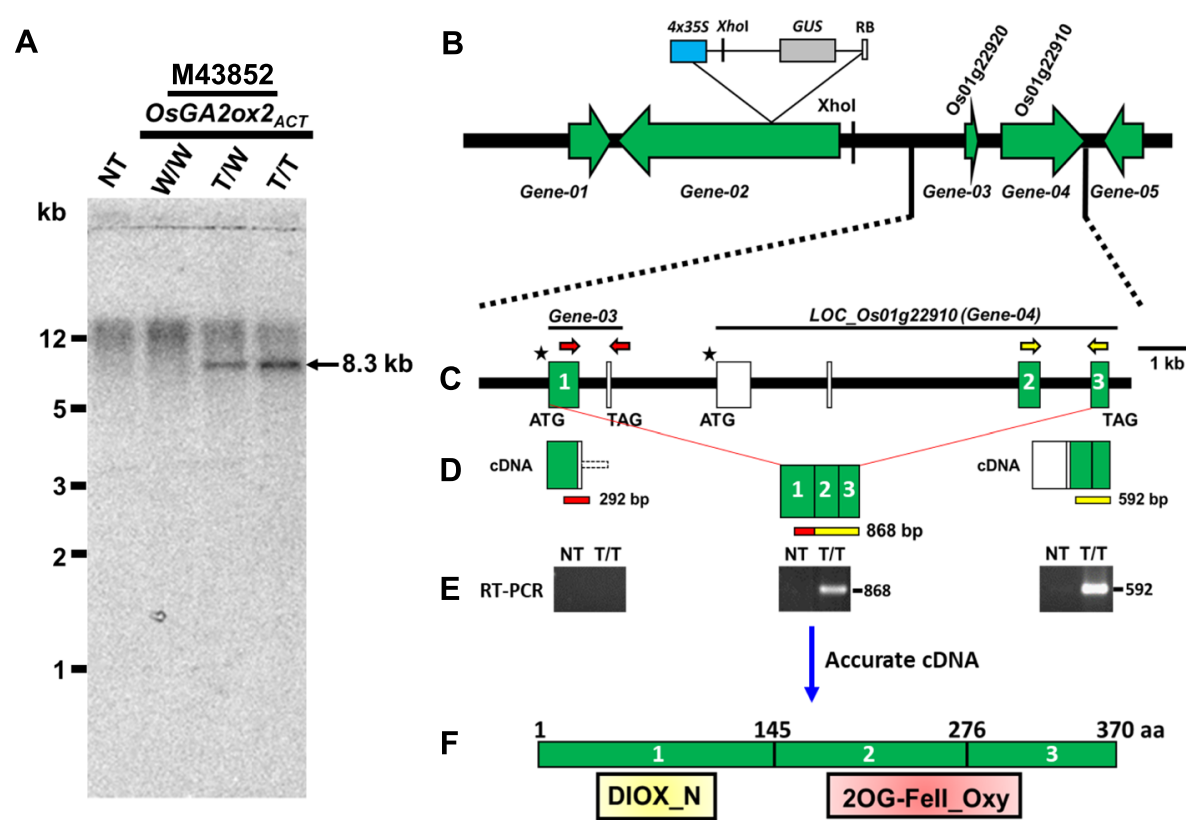

Fig. 2 Southern blot assay of mutant M43852 and a schematic illustration of the RT-PCR results and possible CDNA and exon/intron structures to reannotate the gene structure of OsGA20x2. a Southern blot assay showing only one copy of the T-DNA insertion in mutant M43852. b A schematic diagram showing five genes (named gene-01 to gene-05) annotated in a $40 \mathrm{~kb}$ region flanking the T-DNA insertion site. c Enlarged regions of annotated gene-03 (LOC_Os01g22920) and gene-04 (LOC_Os01g22910) with their possible exon (box) and intron (bold line) structures. The two primer sets (arrows) utilized to detect gene expression are shown above their genes. $\mathbf{d}$ CDNAs and expected sizes of RT-PCR products using the corresponding primer sets for the annotated gene-03, gene-04 and reannotated gene OsGA20x2. e RT-PCR results of gene-03 (using the red primer set), gene-04 (using the yellow primer set that amplify $592 \mathrm{bp}$ ) and the reannotated gene OsGA2ox2 (using the 5' red and 3' yellow primer sets that amplify $868 \mathrm{bp}$ ). $\mathbf{f}$ Accurate cDNAs for the reannotated OsGA20x2. Three exons with 370 amino acids and the relative locations of the DIOX_N and 2OG-Fell_Oxy conserved protein domains

plant height (Fig. 1b). Although LOC_Os01g22910 was annotated as a retrotransposon protein (Kawahara et al. 2013), its deduced protein contained a 2OG-FeII_Oxy domain, one of the conserved domains of GA2ox (Fig. 2f). GA 2-oxidase belongs to the DOXC class of the 2OGD superfamily, whose members contain a conserved DIOX_N domain (Pfam ID: PF14226) at the N-terminal region and a 2OG-FeII_Oxy domain (Pfam ID: PF03171) at the carboxyl terminus (Kawai et al. 2014). The deduced protein of LOC_Os01g22920 (gene-03, putative OsGA2ox2) contains a DIOX_N domain but no 2OGFeII_Oxy domain in its C-terminal region (Han and Zhu 2011; Liu et al. 2018), and its downstream gene LOC_ Os01g22910 contains a 2OG-FeII_Oxy domain, implying that LOC_Os01g22910 is part of OsGA2ox2.

To verify the above implications, RT-PCR analysis for mutant M43852 with various primer combinations was performed. While an expected $292 \mathrm{bp}$ signal (Fig. 2d) with a primer set based on the gene-03 annotation (Fig. 2c) was not detected (Fig. 2e, the same RT-PCR result shown in Fig. 1b), the 592 bp signal with a primer set based on the gene- 04 annotation and the $868 \mathrm{bp}$ signal (Fig. 2d) with a primer set for exons \#1 and \#3 of the new annotation, including a combination of gene-03 and gene-04 with three misannotated exons (empty boxes) as part of the first long $\sim 8 \mathrm{~kb}$ intron (Fig. 2c), was strongly activated (Fig. 2e). With these RT-PCR results, we reannotated this OsGA2ox2 gene and proposed that this OsGA2ox2 gene is the combination of LOC Os01g22920 and LOC_Os01g22910, with three exons (green boxes) and a long first intron (Fig. 2c). This annotation was further confirmed by an OsGA2ox2 cDNA clone isolated from the OsGA2ox2-activated T-DNA mutant M43852 and functionally identified by overexpressing OsGA2ox2 in transgenic rice plants (Fig. 3c). We therefore concluded that this OsGA2ox2 gene contains a long first intron and three exons and encodes 370 amino acids, with two typical conserved DIOX_N and 2OG-FeII_Oxy protein domains (Fig. 2c, f), and the gene is functional.

\section{Functional Identification of Rice GA2ox1, GA2ox2, GA2ox4, GA20x7 and GA2ox8 with Overexpression Transgenic} Plants

In the rice GA2ox gene family, OsGA2ox3 and OsGA2ox 5,6 , and $9\left(\mathrm{C}_{20}\right.$-type GA2ox genes) were characterized in our previous study by overexpressing them in transgenic plants (Lo et al. 2008). To complete the functional identification of all the members of the rice GA2ox gene family, the full-length cDNAs of the other OsGA2ox 

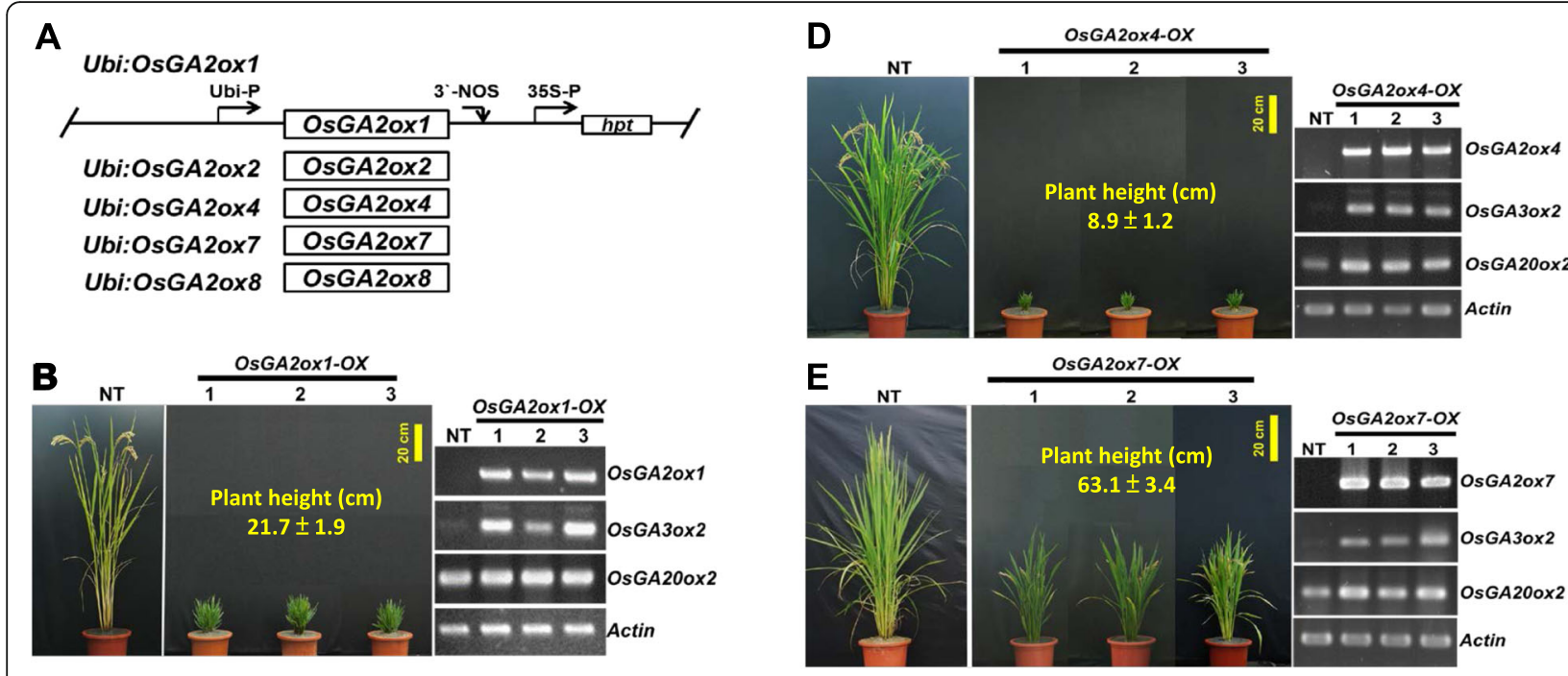

OSGA2OX7-OX
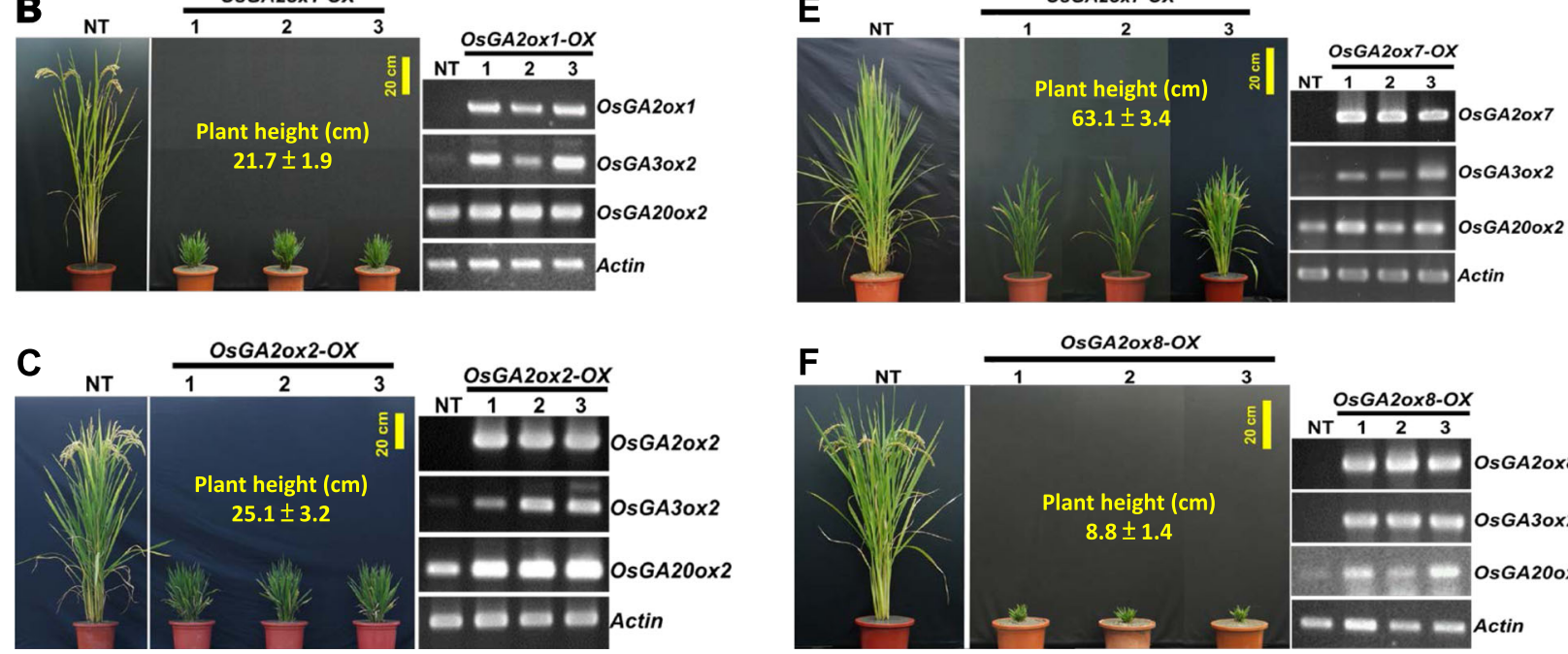

OSGA2OX8-OX

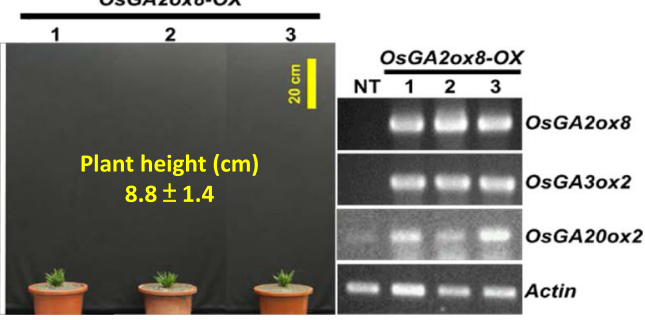

Fig. 3 Phenotypic comparisons and characterization of OsGA20x1, 2, 4, 7, and 8 expressions in overexpression transgenic rice plants. a Schematic diagram showing the overexpression constructs of Ubi:OsGA2ox1, Ubi:OsGA20x2, Ubi:OsGA2ox4, Ubi:OsGA2ox7, and Ubi:OsGA20x8. The full-length CDNA from each gene was cloned into the same vector construct. Three representative $T_{0}$ transgenic plants from each of the OsGA2ox $1-0 X$ (b), OsGA20x2-OX (c), OsGA2OX4-OX (d), OsGA2ox7-OX (e), and OsGA2OX8-OX (f) transgenic lines were compared to their nontransgenic (NT) plants. The average plant height in $\mathrm{cm}$ (means $\pm \mathrm{SE}, \mathrm{n} \geqq 10$ ) for each gene constructs are shown. RT-PCR was performed to analyze the respective target genes and marker genes of OsGA30x2 and OsGA200x2, and the Actin gene (as an internal control) is shown

genes, including GA2ox1, GA2ox2, GA2ox4, GA2ox7 and GA2ox8, were cloned from their respective T-DNA activation-tagged (OsGA2oxs $\mathrm{ACT}_{\text {) }}$ mutants (Fig. 1) and expressed ectopically in TNG67 driven by the maize ubiquitin (Ubi) promoter construct Ubi:OsGA2oxs (Fig. 3a), the same vector used in all our previous studies (Lo et al. 2008; Lo et al. 2017).

Three independent transgenic rice lines with each $U b i$ : OsGA2ox transformation were generated, and the RNA expression of their target OsGA2ox genes was visualized (Fig. 3b-f, and the top row of each gel). As expected, dwarf phenotypes were observed for all OsGA2ox overexpression (OsGA2oxs-OX) transgenic plants. OsGA2ox1-OX and OsGA2ox2-OX showed moderate reductions (Fig. 3b, c), OsGA2ox4-OX and OsGA2ox8-OX showed severe reductions (Fig. 3d, f), and OsGA2ox7-OX showed minor reductions (Fig. 3e) in height. These varying degrees of plant height reduction suggested that the GA deactivation capability varied. To explore whether the plant height reduction in OsGA2oxs-OX transgenic plants resulted from GA deficiency, the expression of two GA deficiency-regulated biosynthetic genes,
OsGA3ox2 and OsGA20ox2, was investigated, and the expression of both of them was enhanced in all examined OsGA2oxs-OX transgenic rice lines (Fig. 3b-f, the middle two rows of the gel). In addition, the dwarf seedlings germinated from the two seed-bearing lines (OsGA2ox2-OX and OsGA2ox7-OX), and their phenotype could be rescued by exogenous $\mathrm{GA}_{3}$ treatment (Additional file: Fig. S2).

\section{OsGA20x7-OX Transgenic Rice Plants Revealed a Distinct} Semidwarf Phenotype Different from that of the Other OsGA2oxs-OX Transgenic Plants

To verify whether each member of the OsGA2ox family showed various GA deactivation capabilities, transgenic plants overexpressing each OsGA2ox gene ectopically in the TNG67 rice variety with the same vector construct were obtained and pooled together for comparison (Fig. 4a). The OsGA2ox overexpression transgenic plants in class II and class III presented approximately $12.1 \%$ to $30.9 \%$ RPH compared with that of the nontransgenic (NT) plants. Unlike the severe dwarf with approximately $8.3 \%$ to $8.7 \% \mathrm{RPH}$ caused by the OsGA2ox3, 4 and 8 


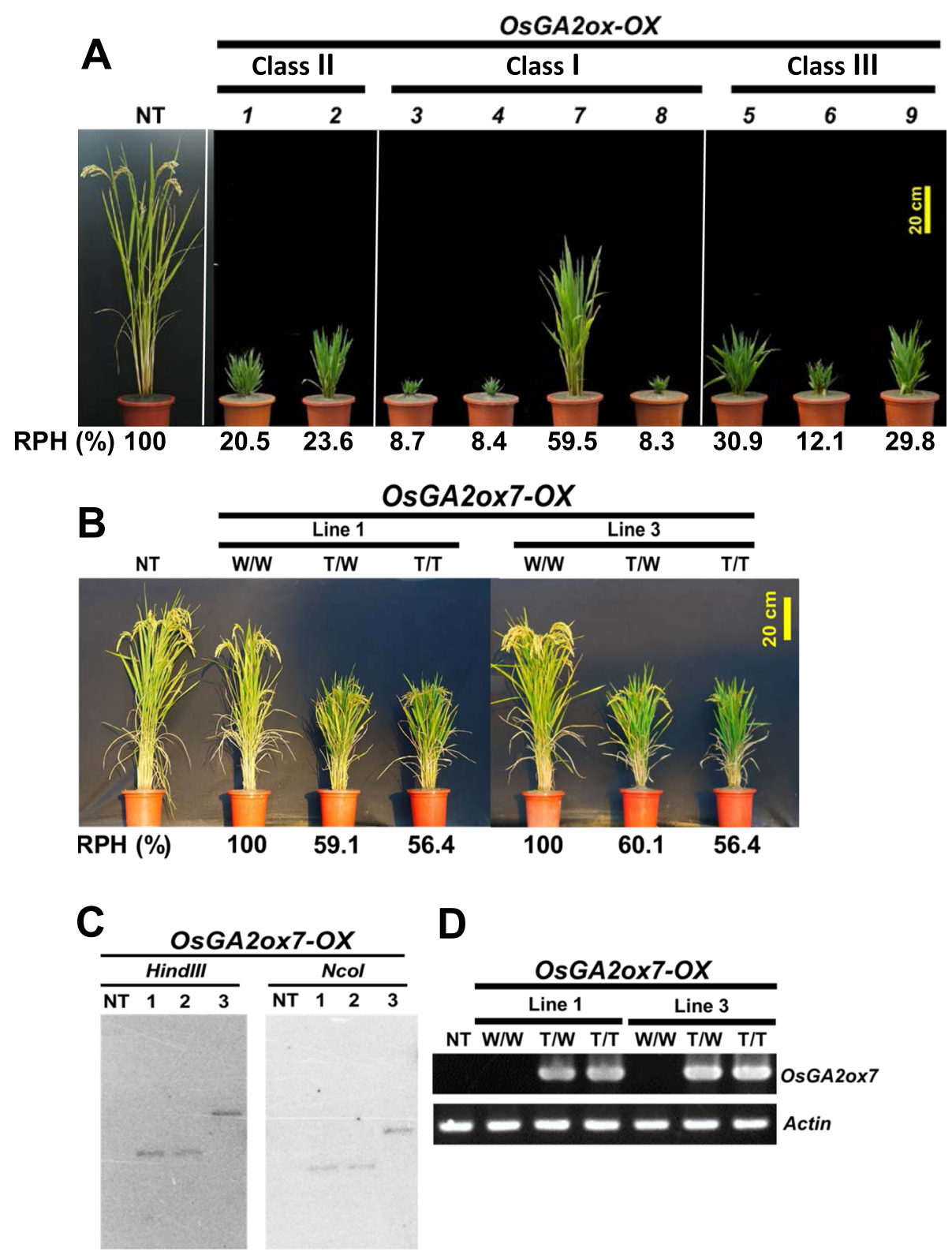

Fig. 4 Phenotypic comparison of the transgenic rice plants overexpressing all the rice GA20x genes and characterization of the OsGA20x7-OX transgenic lines. a Phenotypic comparison of the transgenic rice plants overexpressing for all rice GA2ox genes. A representative $T_{0}$ transgenic plant overexpressing each of the 9 rice GA2ox genes under the same comparison basis is shown. The plants were grown in a greenhouse. $\mathbf{b}$ Phenotypic comparison of the OsGA2OX7-OX overexpression transgenic lines. The insertion events of lines 1 and 3 were identified, and their phenotypes vs genotypes (W/W no T-DNA; T/W hetero; T/T homo) and RPH compared with that of the NT plants are shown. The plants were grown in paddy fields. c Southern blot assay showing a single T-DNA insertion for all three lines. The line-1 and line-3 showing independent insertion events. $\mathbf{d}$ Results of RT-PCR analysis of various genotypes of OsGA20X7-OX transgenic line-1 and line-3. The Actin gene was used as a control

genes, the OsGA20x7-OX in the same class (I) resulted in approximately $59.5 \% \mathrm{RPH}$ (Fig. 4a), which garnered our attention for further investigation.

These distinct semidwarf OsGA2ox7-OX transgenic plants were further evaluated with two independent lines (Fig. 4b), each with one copy of a T-DNA insertion confirmed by Southern blotting assays (Fig. 4c). The T-
DNA that inserted into the long arm of chromosome \#12 for line-1 and into the short arm of chromosome \#3 for line-3 were identified, and the RPH (\%) of their corresponding genotypes (Fig. 4b), along with high levels of OsGA2ox7 expression, were stably inherited in their progeny (Fig. 4d). The agronomic trait data of the transgenic plants, compared with the NT plants, presented 
approximately 59-61\% RPH for heterozygous (T/W) plants, $55-56 \% \mathrm{RPH}$ for homozygous $(\mathrm{T} / \mathrm{T})$ plants, a $30 \%$ increase in tiller number and a panicle length of approximately $80 \%$ during the spring 2020 season (Table 1).

\section{Phylogenetic Analysis of Class I GA2ox Genes in the Poaceae Revealed Two Functionally Distinct Clades}

The plant height-reducing effect of $O s G A 2 o x 7$ was much weaker than that of other members in class I, which led us to compare whether any variation existed among class I genes that might cause this difference. The sequences of all available GA2ox genes that were categorized into class I were collected and analyzed. A phylogenetic tree comprising class I GA2ox genes from Brachypodium distachyon (Bd), Oryza sativa (Os), Setaria italica (Si), Sorghum bicolor (Sb), Spirodela polyrhiza (Sp) and Zostera marina $(\mathrm{Zm})$ was generated. In the phylogenetic tree, the class I GA2oxs of Poaceae species could be further divided into clade A and clade B (Fig. 5a). The $Z m G A 2 o x s$ and SpGA2oxs were not included in either clade A or clade B, suggesting that both clades formed after the divergence of the early branching of monocots but before Poaceae species diverged. Clade B included orthologs of OsGA2ox7 and OsGA2ox10; however, OsGA2ox10 was excluded from the tree due to its absence of the DIOX_N domain (Fig. 5a).

Unlike in clade $B$ genes, overexpression of clade A genes (OsGA2ox3, 4 and 8) caused a significant effect on growth suppression of transgenic rice plants (Fig. 4a). To further confirm whether the growth suppression effect was different between clade A and clade B, orthologs of OsGA2ox3 (Bd2g50280), OsGA2ox4 (Bd2g19900), OsGA2ox7 (Bd2g06670), and OsGA2ox10 (Bd2g32577) from B.distachyon were ectopically overexpressed in transgenic rice plants. Three independent lines revealed the same RNA expression levels from each BdGA2oxs$O X$ transgenic rice plant were compared, and $B d 2 g 50280-O X$ and Bd2g19900-OX showed severe dwarf phenotypes (Fig. 5b) similar to those resulting from the rice clade A genes (Fig. 4a), while $B d 2 g 06670-O X$ and $B d 2 g 32577-\mathrm{OX}$ transgenic rice plants revealed less growth suppression (Fig. 5b), the trait of which was similar to that caused by OsGA2ox7, a clade B gene (Fig. 4a). Unlike the Bd2g50280-OX and Bd2g19900-OX transgenic rice plants, the $B d 2 g 06670-O X$ and $B d 2 g 32577-O X$ transgenic rice plants produced viable seeds. This distinct functional effect on growth suppression of plants between clades A and B (Fig. 5c) within class I suggests that the GA deactivation capability may have been attenuated in clade $B$, which might have been caused by certain amino acid variations or structural differences in the members between these two clades.

\section{Four Amino Acid Variants in OsGA2ox3 Attenuated its Capability for GA Deactivation}

To determine which amino acids might be responsible for the different GA deactivation capabilities between members of clades A and B of class I GA2oxs, the amino acid sequences of class I GA2oxs from Poaceae species were first analyzed by the MEME algorithm to identify conserved protein motifs (Bailey et al. 2015). A total of 15 conserved motifs were identified (Fig. 6a), and these motifs were further aligned to identify whether any significant amino acid variations occurred between clades $\mathrm{A}$ and $\mathrm{B}$. Two distinguishable amino acid variants were revealed by sequence alignment comparing available sequences of clade A and B genes from O. sativa (Os), B. distachyon $(B d)$, S. bicolor $(S b)$ and $S$. italica (Si) (Fig. $6 \mathrm{~b}, \mathrm{c})$. The first variant is $\mathrm{Gln}(\mathrm{Q} 220)$ in clade A vs Glu (E227) in clade B in motif 10 (Fig. 6b), and the second variant is Tyr (Y274) in clade A vs Phe (F283) in clade B in motif 13 (Fig. 6c); they were positioned near the iron (HTD)- and the 2-oxoglutarate (2OG)-binding sites (RVS) (Fig. 6b, c). A 3-D protein structure of OsGA2ox3 with accession number 6KU3 downloaded from the Protein Data Bank was used to show the relative positions of amino acids Q220 and Y274 in the catalytic pocket (Additional file: Fig. S3).

To investigate whether these two amino acids were important and could attenuate the GA deactivation capability, the amino acids Q220 and Y274 in OsGA2ox3 were replaced with glutamic acid (E) and phenylalanine (F), respectively, or simultaneously to create OsGA2ox3E

Table 1 Agronomic traits comparisons between OsGA20X7-OX transgenic lines and NT/TNG67 plants"

\begin{tabular}{|c|c|c|c|c|c|c|c|}
\hline \multicolumn{2}{|c|}{ Rice plants } & Plant height (cm) & Heading date (DAI) & Tiller number & Panicle number & Panicle length $(\mathrm{cm})$ & 1000 -grain weight $(\mathrm{g})$ \\
\hline \multicolumn{2}{|c|}{ NT/TNG67 } & $114.0 \pm 4.6^{a}$ & $115.2 \pm 1.2^{\mathrm{a}}$ & $14.6 \pm 1.1^{a}$ & $14.0 \pm 2.0^{\mathrm{a}}$ & $16.5 \pm 1.2^{\mathrm{a}}$ & $25.4 \pm 0.6^{a}$ \\
\hline \multirow[t]{2}{*}{ Line-1 } & $\mathrm{T} / \mathrm{W}$ & $65.2 \pm 1.8^{\mathrm{b}}$ & $117.8 \pm 1.3^{b}$ & $19.4 \pm 1.8^{b}$ & $18.2 \pm 2.9^{b}$ & $13.5 \pm 0.7^{b}$ & $23.3 \pm 0.8^{b}$ \\
\hline & $\mathrm{T} / \mathrm{T}$ & $61.6 \pm 2.1^{c}$ & $118.1 \pm 1.1^{b}$ & $19.6 \pm 2.7^{b}$ & $18.6 \pm 2.4^{b}$ & $13.4 \pm 0.5^{\mathrm{b}}$ & $22.8 \pm 1.0^{b}$ \\
\hline \multirow[t]{2}{*}{ Line-3 } & $\mathrm{T} / \mathrm{W}$ & $67.8 \pm 1.3^{b}$ & $117.4 \pm 1.2^{\mathrm{b}}$ & $19.4 \pm 2.9^{b}$ & $19.0 \pm 2.7^{b}$ & $13.5 \pm 0.5^{\mathrm{b}}$ & $22.6 \pm 1.1^{b}$ \\
\hline & $\mathrm{T} / \mathrm{T}$ & $62.4 \pm 3.3^{c}$ & $118.3 \pm 1.3^{b}$ & $20.2 \pm 2.8^{\mathrm{b}}$ & $19.6 \pm 2.7^{b}$ & $14.3 \pm 0.7^{b}$ & $23.5 \pm 0.6^{\mathrm{b}}$ \\
\hline
\end{tabular}

The different letters indicate significant differences between samples according to the Turkey's HSD test $(p<0.05)$. NT/TNG67: rice variety TNG67; T/W heterozygous, T/T homozygous for transgene

$D A l$ days after imbibition

\#The values are the means \pm SEs $(n=5)$ 


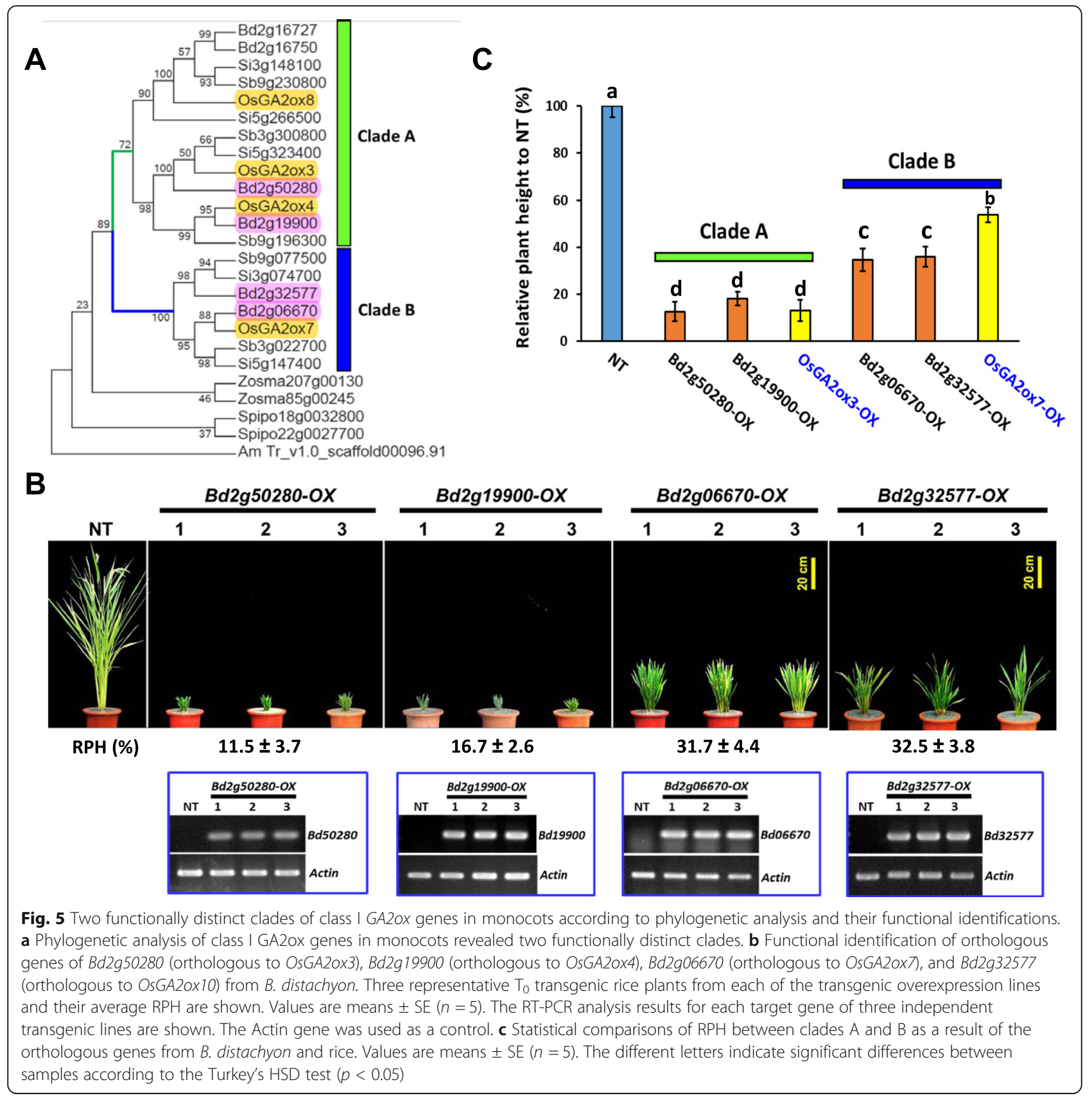

(3E), OsGA2ox3F (3F) and OsGA2ox3EF (3EF) mutants. These 3E, 3F, and 3EF mutant and wild-type (WT) OsGA2ox3 genes were expressed ectopically in transgenic rice with similar high RNA expressions (Additional file: Fig. S4) and were used for comparison. The overexpression transgenic plants $3 E-O X$ and $3 F-O X$ showed the same severe dwarf phenotype as their WT-OX transgenic plants did (Fig. 7a). Although some 3F-OX transgenic plants were slightly taller, their height was not significantly different among the population. However, the double point mutated gene in the $3 E F-O X$ transgenic plants caused a significant plant height increase (Fig. 7a).
Unlike the severely dwarf $3 E-O X, 3 F-O X$ and WT-OX transgenic plants, which showed no panicles, the $3 E F$ $O X$ transgenic plants showed protruding panicles (Fig. $7 \mathrm{~b}, \mathrm{c})$ and produced some viable seeds. The culms were dissected to show that the relative length of the panicle and internodes (Fig. $7 \mathrm{~d}$ ) and the length of the panicle and all internodes in $3 E F-O X$ transgenic plants all increased (Fig. 7e), suggesting that the replacement of Q220E and Y274F in OsGA2ox3 attenuated its GA deactivation capability.

Since the $3 E F-O X$ plants presented only approximately $24 \% \mathrm{RPH}$ compared with that of the NT plants (Fig. 7a), 

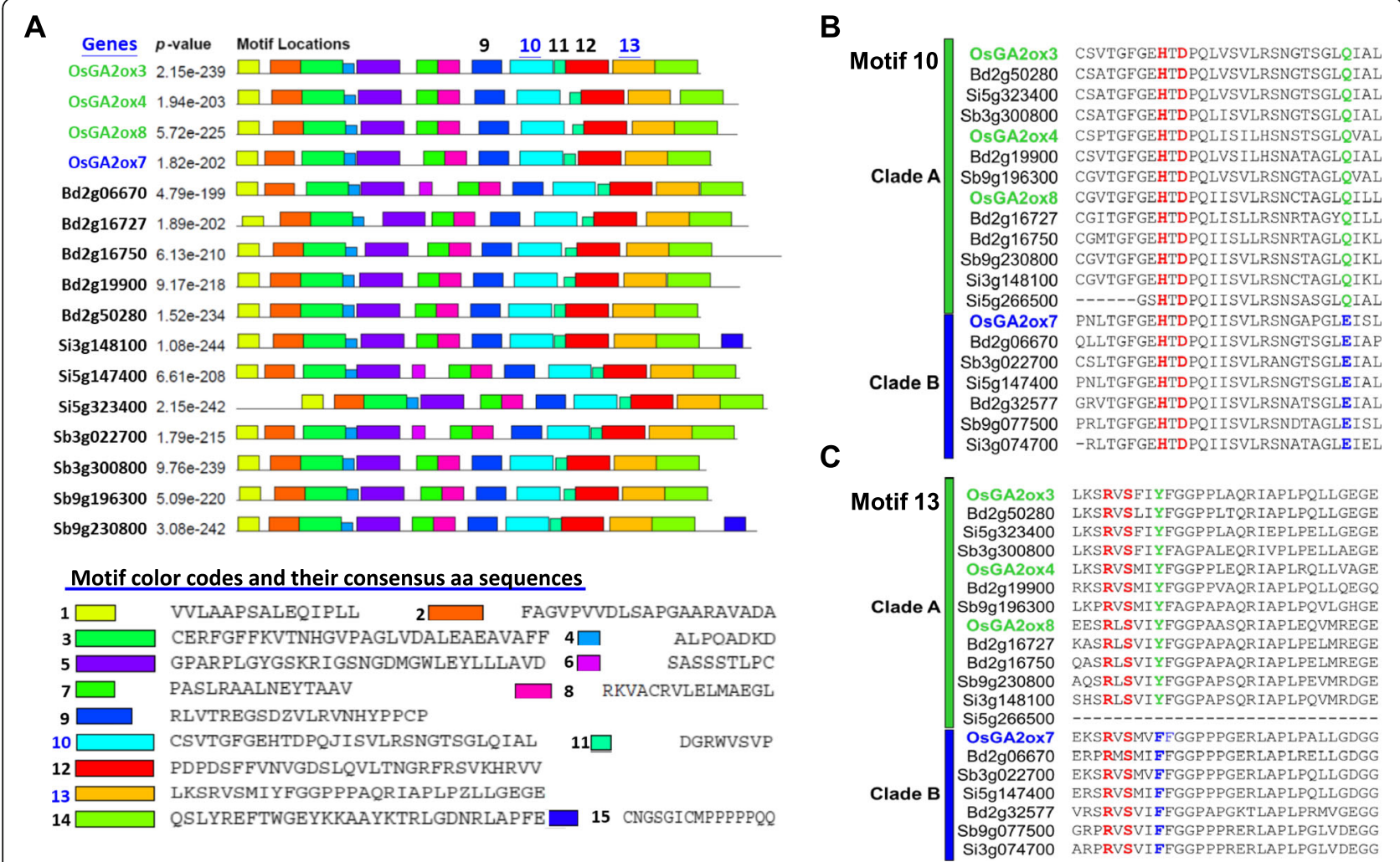

Fig. 6 Conserved protein motif identification among class I GA2oxs from Poaceae species and identification of distinguishable amino acids between clades A and B. a Conserved protein motif identification among 16 class I GA2oxs from Poaceae species by MEME algorithm analysis. The alignment of the 15 conserved motifs indicated by color codes and the consensus amino acid sequences of each conserved motif are shown. $\mathbf{b}$ Detailed consensus amino acid sequence alignment of motif 10, distinguished by $Q$ in clade A and E in clade B. A putative iron-binding site (HTD) is shown in red. c Detailed consensus amino acid sequence alignment of motif 13, distinguished by $Y$ in clade A and F in clade B. A putative 2-OG-binding site is shown in red

which was not comparable to the approximately $60 \%$ $\mathrm{RPH}$ of the NT plants observed for OsGA2ox7-OX (Fig. 4a), this suggests that the attenuation of the GA deactivation capability of OsGA2ox7 could not be fully determined by the replacement of Q220 and Y274. To further investigate any other causal amino acid variants involved in attenuating GA deactivation capability, the protein structure of OsGA2ox7 was modeled by using the Phyre2 server (Kelley et al. 2015) and compared to the structure of OsGA2ox3 (Fig. 8a). A loop region that contains approximately 13-14 amino acids located between motif 9 and motif 10 showed a divergent structure between OsGA2ox3 and OsGA2ox7 (Fig. 8a, b). Sequence alignment between clades A and B revealed two distinguishable amino acid variants: a variant Cys (C186) in OsGA2ox3 vs Arg (R191) in OsGA2ox7 and another variant Cys (C194) in OsGA2ox3 vs Pro (P201) in OsGA2ox7 in this loop region (Fig. 8a).

To evaluate the impact of these amino acid variants on GA2ox activity, amino acids C186 and C194 of OsGA2ox3 were replaced with arginine $(\mathrm{R})$ and proline $(\mathrm{P})$, respectively, or simultaneously to create OsGA2ox3R (3R),
OsGA2ox3P (3P) and OsGA2ox3RP (3RP) mutants. The transgenic plants overexpressed these mutated genes all at the same high levels (Additional file: Fig. S5). The transgenic plants overexpressing the $3 \mathrm{P}$ mutant $(3 \mathrm{P}-\mathrm{OX})$ showed a slight plant height increase from 13\% to approximately $17 \% \mathrm{RPH}$ of that of the NT plants. However, $3 R-O X$ transgenic plants showed a greater $\mathrm{RPH}$ increase of up to approximately $29 \%$ of that of the NT plants, and the double point-mutated gene in the $3 R P-O X$ transgenic plants resulted in a significant RPH increase of up to approximately $40 \%$ to $47 \%$ of that of the NT plants (Fig. 8c). The 3R-OX and 3RP-OX transgenic plants showed protruding panicles (Fig. 8c) and could produce viable seeds, with increased panicle and internode lengths (Fig. 8d). These observations suggested that the replacement of C186R and C194P in the loop region of OsGA2ox3 attenuated its GA deactivation capability.

\section{Analysis of Gene Structure and Sequence Differences Suggest that OsGA2ox10 is a Pseudogene}

The genomic structures of nine functionally confirmed (OsGA2ox1 to 9) and annotated (OsGA2ox10) 

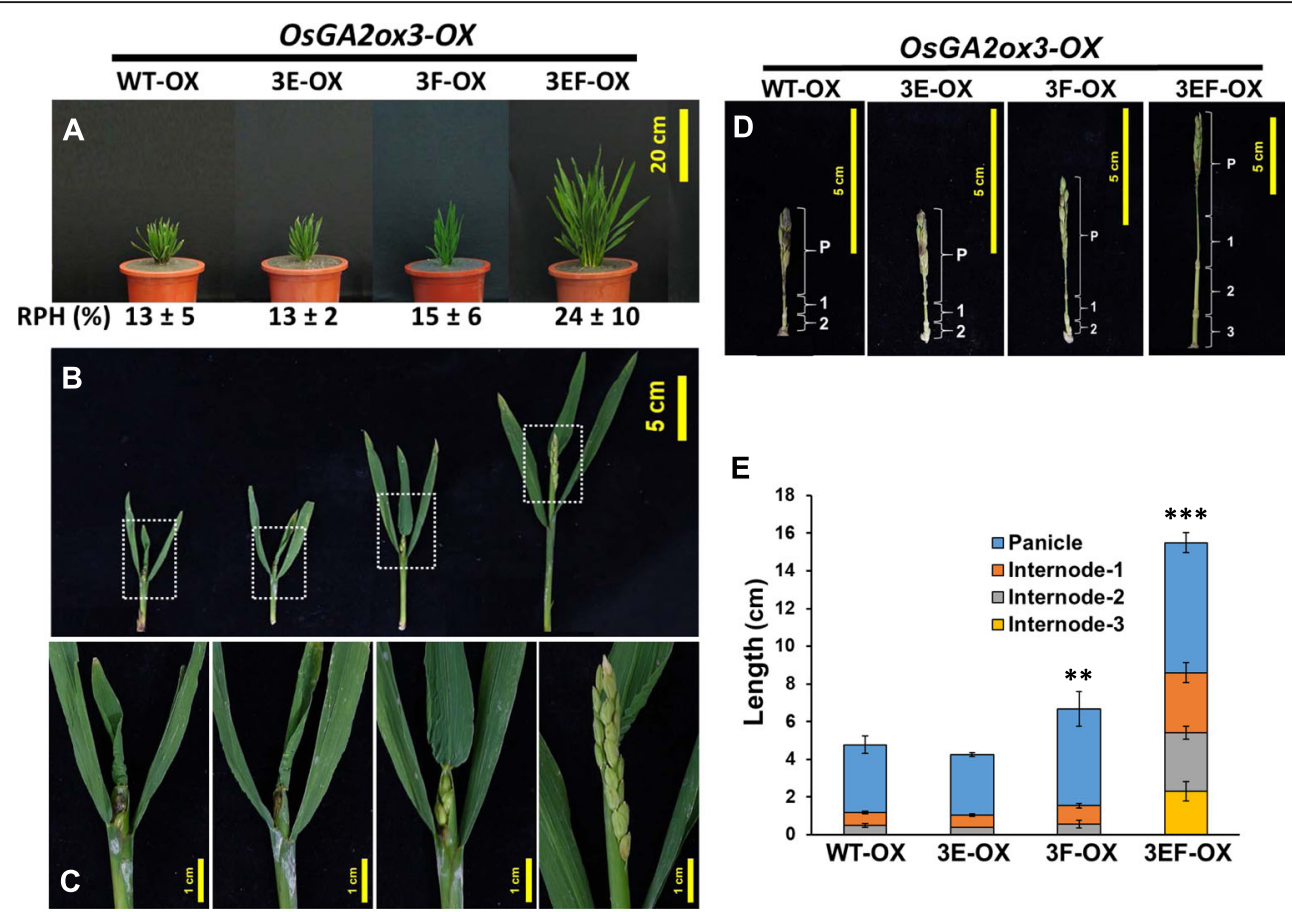

Fig. 7 Phenotypic comparison of WT-OX, 3E-OX, 3F-OX and 3EF-OX transgenic rice plants. a Phenotypic comparison of WT-OX, 3E-OX, 3F-OX and $3 \mathrm{EF}-\mathrm{OX}$ transgenic rice plants. A representative $\mathrm{T}_{0}$ transgenic plant with the average $\mathrm{RPH}$ resulting from each overexpression line is shown. Values are means $\pm \mathrm{SE}(n=5)$. $\mathbf{b}$ A dissected representative main culm from each overexpression plant is shown to compare their emerging panicles at the maturation stage. $\mathbf{c}$ Enlarged image from the boxed area of B) to show details. $\mathbf{d}$ Comparisons of the length of panicles and internodes from each overexpression line. P: panicle; 1: internode \#1; 2: internode \#2; 3: internode \#3. e Statistical comparisons of the length of the main culms (including panicles and internodes) from each overexpression line. Values are means $\pm \operatorname{SE}(n=5)$. The significant differences using Student's $t$-test are indicated by ${ }^{*} p<0.05,{ }^{* *} p<0.01,{ }^{* * *} p<0.001$

genes were compared. The majority of OsGA2oxs contained three exons and two introns; however, OsGA2ox4 and OsGA2ox8 contained two exons, and OsGA2ox5 contained a single exon (Fig. 9a). Based on sequence comparison, the large exon 2 of OsGA2ox4 could have resulted from the fusion of exons 2 and 3; the large exon 1 of $O s G A 20 x 8$ could have resulted from the fusion of exons 1 and 2; and the single exon of OsGA2ox5 could have resulted from the fusion of the three exons. Other than the difference in numbers and lengths of the exons, significant intron length variations among them were also found. First, class II genes have the longest first intron range from 4 to 8 $\mathrm{kb}$; second, class I OsGA2oxs could be divided into a relatively long first intron, such as that in OsGA2ox7 and OsGA2ox10, and a very short first intron, such as that in OsGA2ox3 and OsGA2ox4; and third, OsGA2ox10 had longer first intron, a truncated first exon and an extended last exon (Fig. 9a).

In addition to genomic structure analysis, genomic sequence variation analysis of all OsGA2oxs among 4276 rice accessions (Zhao et al. 2015) was performed. Single-nucleotide polymorphisms (SNPs) and insertions/deletions (INDELs) in $5^{\prime}$-regulatory regions and INDELs and SNPs including nonsynonymous mutations (dNs) and synonymous mutations (dSs) in coding regions were collected from the RiceVarMap database (Zhao et al. 2015). The numbers of $\mathrm{dN}$-, dS- and INDEL-causing frameshift mutations in the coding regions were calculated and compared (Table 2). The annotated OsGA2ox10 gene contains $20 \mathrm{dNs}$, which was approximately 3 -fold higher than the average number of $\mathrm{dNs}$ found in other OsGA2oxs, and OsGA2ox10 has the highest number of INDEL and frameshift mutations, suggesting that the strength of selection constraints in the coding sequence of OsGA2ox10 was much less than that in other OsGA2ox genes. In addition, OsGA2ox10 contained no DIOX_N domain, which was different from the orthologs of other grass species that contained both DIOX_N and 2OG-FeII_Oxy conserved domains (Fig. 9b). In addition, transgenic rice overexpressing one B. distachyon ortholog of OsGA2ox10, $B d 2 g 32577$, which contains both conserved domains, showed a functional dwarf phenotype (Fig. 5b). With the evidence of high mutation rates in the coding region and abnormal gene structure, plus the nonexpression of OsGA2ox10 observed in previous studies 


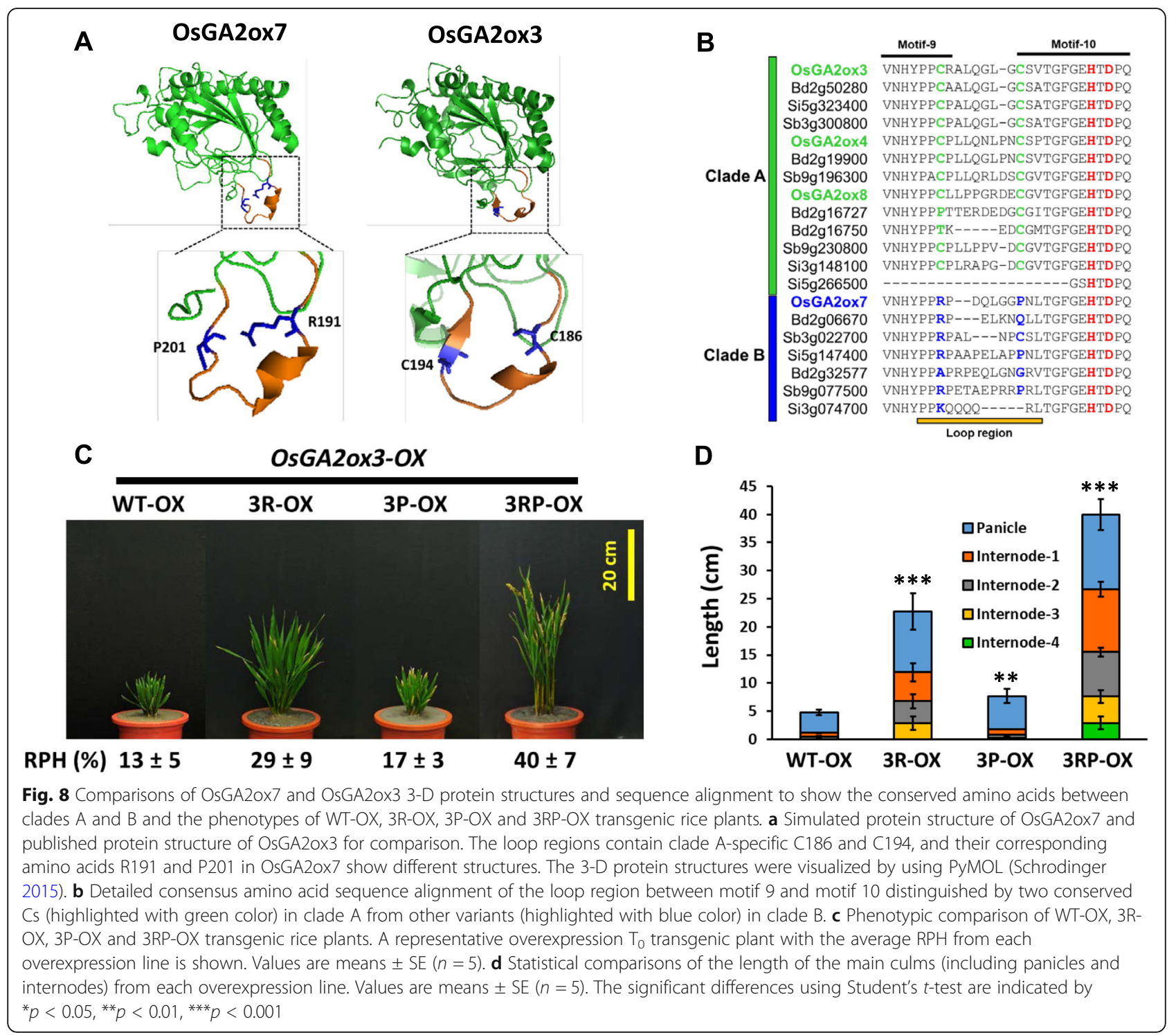

(Lo et al. 2008; Hirose et al. 2012), we inferred that OsGA2ox10 is a pseudogene.

\section{Discussion}

\section{The Rice GA2ox Gene Family Comprises Nine Functional} GA2oxs

Based on phylogenetic analysis, the existence of 10 rice GA2ox genes has been proposed, and out of these 10 genes, OsGA2ox10 has been suggested to be a pseudogene (Lo et al. 2008). This is due to its irregular gene structure caused by the truncation of the first exon and extension of the last exon (Fig. 9a, b), and RNA expression of this gene is not detected in all analyzed tissues (Lo et al. 2008; Hirose et al. 2012). From genomic sequence variation analysis of OsGA2oxs among 4276 rice accessions, OsGA2ox10 revealed the highest number of $\mathrm{dN}$, INDEL and frameshift mutations in its coding region (Table 2), indicating that OsGA2ox10 is the least stringent among OsGA2oxs under selective constraints. In addition, while transgenic rice overexpressing the ortholog Bd2g32577 of OsGA2ox10 from B. distachyon, which contains two typical conserved domains, showed a functional dwarf phenotype (Fig. 5b), no plant heightreducing effect was observed in the transgenic plants overexpressing synthetic OsGA2ox10 cDNA (data not shown). We therefore conclude that OsGA2ox10 is a pseudogene.

Other than the findings concerning OsGA2ox10, the present study demonstrated that five (OsGA2ox1, $2,4,7$, and 8) additional rice GA2ox genes are functional in TNG67 based on the results of the T-DNA activation-tagged mutants (Fig. 1) and the ectopically overexpressed transgenic plants (Fig. 3). Although some T-DNA activation-tagged mutants such as 


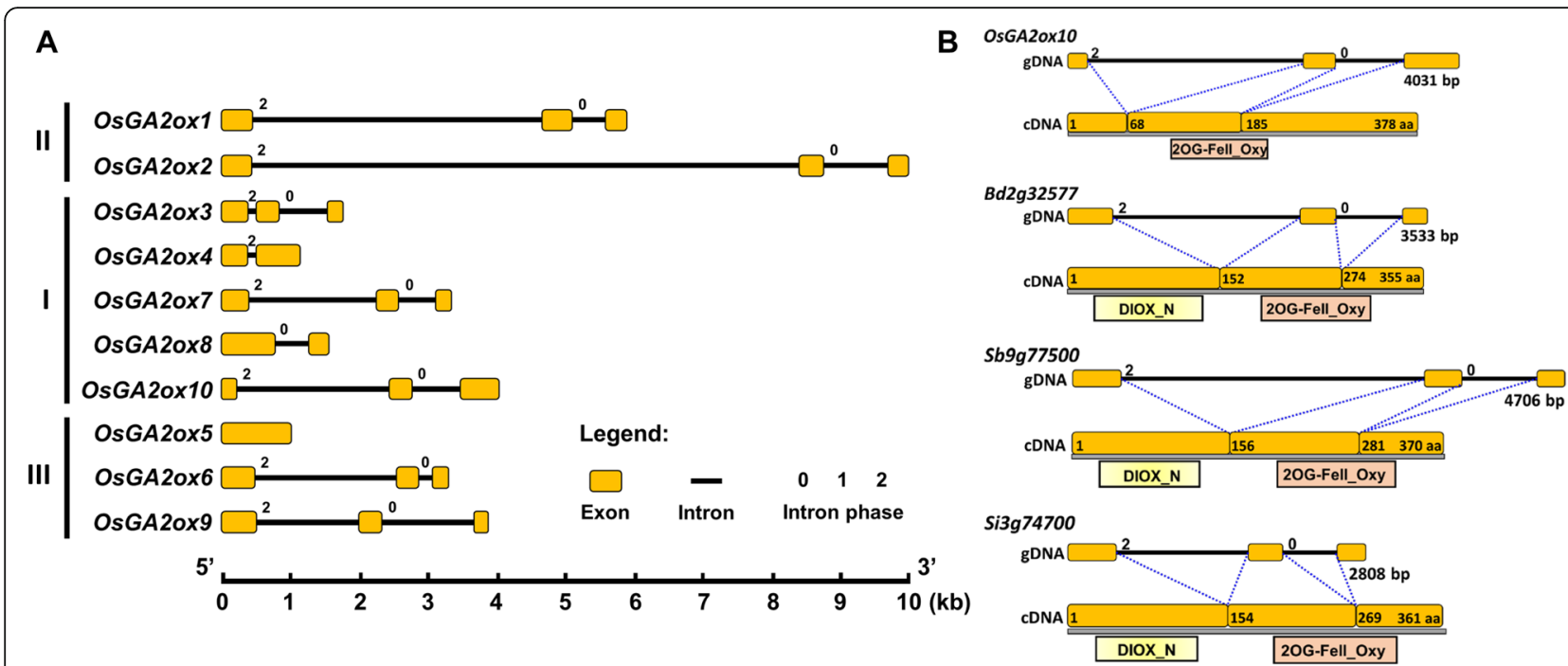

Fig. 9 Gene structure of the rice GA20x family members and comparison of the functional domains in OsGA20x10 and its orthologous genes. a Gene structures of all 10 rice GA2ox genes. The sizes of exons (box) and introns (line) are shown on the same scale. The numbers on top of each exon/intron junction indicate their intron phases. $\mathbf{b}$ Schematic diagram showing the exon-intron gene structure and amino acids encoded by the respective exons in OsGA20x10, Bd2932577, S69g 77500 and Si3g74700. The corresponding regions of the DIOX_N and 2OG-Fell_Oxy functional domains are presented below the exons, and DIOX_N and 2OG-Fell_Oxy are represented by yellow boxes and light pink boxes, respectively

M36548 for OsGA2ox1 and M66925 for OsGA2ox7 did not show a significant plant height reduction, the overexpression transgenic plants for each of these five genes did show significantly reduced height (Fig. 3). In addition, the RNA expression of the GA deficiency-inducible OsGA20ox2 and OsGA3ox2 genes was significantly induced in each of the OsGA2ox overexpression transgenic lines (Fig. 3). With the results of these five functional genes plus the previously identified four (OsGA2ox3, 5, 6, and 9) genes (Lo et al. 2008), we confirmed that the rice genome contains at least nine functional GA2ox genes.

\section{Members of the OsGA2ox Gene Family Exert Various} Effects on Plant Height Reduction, Suggesting that their GA Deactivation Capability Diverged during Evolution Although the plant height-reducing effects of the OsGA2ox2, OsGA2ox4 and OsGA2ox8 genes in T-DNA activation-tagged mutants (Fig. 1) had the same tendency as the effects from the overexpression transgenic approaches (Fig. 3), the reducing effects in the overexpression transgenic plants were much more severe than those in the activation-tagged mutants. This was because the plant height-reducing effects in the activation-tagged mutants can be influenced significantly by their T-DNA insertion events/locations (Liao et al. 2019) or by

Table 2 Genomic sequence variations in the 5'-regulatory and coding regions of OsGA2oxs analyzed with 4276 rice accessions

\begin{tabular}{|c|c|c|c|c|c|}
\hline \multirow[t]{2}{*}{ Classes } & \multirow[t]{2}{*}{ Genes } & \multirow{2}{*}{$\begin{array}{l}5^{\prime} \text {-Regulatory region } \\
\text { SNP/INDEL }^{b}\end{array}$} & \multicolumn{3}{|c|}{ Coding region } \\
\hline & & & $\overline{\mathrm{dN}}$ & dS & $\overline{F S / I N D E L ~}{ }^{c}$ \\
\hline \multirow[t]{2}{*}{ I } & OsGA2ox1 & $9 / 3$ & 3 & 4 & $0 / 0$ \\
\hline & OsGA2ox2 & $47 / 8$ & 9 & 9 & $0 / 1$ \\
\hline \multirow[t]{5}{*}{ II } & OsGA2ox3 & $23 / 7$ & 4 & 4 & $0 / 0$ \\
\hline & OsGA2ox4 & $48 / 22$ & 10 & 5 & $1 / 1$ \\
\hline & OsGA2ox7 & $37 / 1$ & 7 & 5 & $0 / 2$ \\
\hline & OsGA2ox8 & $45 / 9$ & 3 & 0 & $0 / 0$ \\
\hline & OsGA2ox10 & $29 / 5$ & 20 & 12 & $3 / 7$ \\
\hline \multirow[t]{3}{*}{ III } & OsGA2ox5 & $28 / 12$ & 7 & 2 & $1 / 1$ \\
\hline & OsGA2ox6 & $6 / 1$ & 5 & 4 & $0 / 0$ \\
\hline & OsGA20x9 & $62 / 7$ & 10 & 5 & $0 / 2$ \\
\hline
\end{tabular}

\footnotetext{
${ }^{\mathrm{a}}$ Sequences $1 \mathrm{~kb}$ upstream from the start codon were analyzed
}

${ }^{b}$ Numbers of SNPs and INDELs identified using/with the Rice/VarMap database (Zhao et al. 2015)

${ }^{c}$ Numbers of FSs (frameshift mutations) in coding regions caused by INDELs are shown on top of the total numbers of INDELs 
possible epigenomic changes around the T-DNA insertion sites (Jupe et al. 2019), as the mutant M36548 for OsGA2ox1 showed activation of OsGA2ox1 but caused little effect on plant height reduction, such that the GA deactivation capability could not be evaluated correctly solely on the basis of T-DNA activation-tagged mutants. In contrast, the GA deactivation capability of OsGA2oxs could be easily and correctly verified based on their plant height-reducing effects using the overexpression transgenic approach (Lo et al. 2017).

In the present study, transgenic plants overexpressing each of the OsGA2ox genes ectopically in the same setting were compared, and varying degrees of plant height reduction ranging from approximately $8 \%$ to $60 \% \mathrm{RPH}$ were observed (Fig. 4a). Overexpressing each of the class II and class III genes caused dwarf phenotypes with approximately $12 \%$ to $31 \% \mathrm{RPH}$, and overexpressing each of the class I genes caused either severe dwarf ( $8 \%$ to $9 \%$ $\mathrm{RPH}$ for OsGA2ox3, 4, and 8) phenotypes or semidwarf (60\% of RPH for OsGA2ox7) phenotypes (Fig. 4a). These various reducing effects on plant height based on the same comparison suggest that the capability of GA deactivation among rice GA2oxs could have diverged during evolution.

\section{OsGA2ox2 can be Recognized as a Regulatory Hypofunctionalized Gene and is Functionally Preserved in the Rice Genome}

Unlike the Rice Genome Annotation Project (http://rice. plantbiology.msu.edu/), which annotated $L O C_{-}$ OsO1g22920 as OsGA2ox2 (Han and Zhu 2011; Liu et al. 2018), after experimental demonstration, we confirmed that the rice GA2ox2 gene is the combination of $L O C_{-}$ Os01g22920 and LOC_Os01g22910, which includes three exons constituting the typical DOXC class gene in 2OGD (Kawai et al. 2014) and a long first intron (Figs. 2c; 9a). In addition, our annotation for OsGA2ox2 was identical to the annotation of the Os01g0332200 gene from the RAP-DB database (https://rapdb.dna.affrc. go.jp/) (Kawahara et al. 2013; Sakai et al. 2013). Due to the low RNA expression level (Sakai et al. 2003) and large genomic DNA structure of OsGA2ox2, the function of this gene has not been characterized until now.

In the present study, we cloned the full-length cDNA from the T-DNA activation-tagged mutant M43852 and demonstrated that overexpression of OsGA2ox2 has a plant height-reducing effect similar to that of OsGA2ox1 (Figs. 3b, c; 4a). Since OsGA2ox2 and OsGA2ox1 are grouped into the same class II category (Additional file: Fig. S1), are located in the syntenic blocks between chromosomes \#1 and \#5 (Additional file: Fig. S6a, b), and thus have similar exon-intron structures and highly similar amino acid sequences, they were recognized as duplicated genes (Additional file: Figs. S1; S6b). However, their expression profiles were very different, as revealed by comparing the data collected from the Uniformed Viewer for Integrated Omics (UniVIO: http://univio.psc. riken.jp/; Additional file: Fig. S7a; Kudo et al. 2013) database, the Rice Expression Database (RED IC4R: http:// ic4r.org; Additional file: Fig. S7b; Xia et al. 2017) and the Rice Expression Profile Database (RiceXPro; https:// ricexpro.dna.affrc.go.jp/; Additional file: Fig. S7c; Sato et al. 2013). The RNA expression of OsGA2ox2 was barely detectable in all analyzed tissues, and in contrast, the expression level of OsGA2ox1 was significant in reproductive tissues, such as flowers (Additional file: Fig. S7a), panicles and anthers (Additional file: Fig. S7b, c).

Divergence in the expression between duplicated genes is prevalent in plants (Ganko et al. 2007; Li et al. 2009; Renny-Byfield et al. 2014) and might be caused by sequence variations in the cis-regulatory motifs between duplicated genes ( $\mathrm{Li}$ et al. 2005). Phylogenetic footprinting analysis revealed that two regions in the first intron of OsGA2ox1 were highly conserved across the Poaceae (Fig. 10a; Additional file: Table S1a), and a KN1 binding site that was bound by KNOX transcription factor to regulate ZmGA2ox1 expression and that is responsible for the bioactive GA levels around the shoot apical meristem through KNOX protein regulation (Bolduc and Hake 2009) was included in the conserved region (Additional file: Table S2a). Other than the KNOX-binding site, many more conserved binding sites, such as ATHook and GATA binding sites, were also identified in these regions (Additional file: Table S2a). Although the exon-intron gene structure of OsGA2ox2 was similar to that of OsGA2ox1, the first intron of OsGA2ox2 was much longer than that of OsGA2ox1 (Fig. 9a), and the conserved regulatory motifs in OsGA2ox1 were absent in OsGA2ox2 (Fig. 10a). In addition, the number of SNPs/INDELs in the $5^{\prime}$-regulatory region of OsGA2ox2 was approximately 5 times higher than that of OsGA2ox1 (Table 2). This evidence might explain why the expression differed between OsGA2ox1 and OsGA2ox2 (Additional file: Fig. S7). The phenomenon that OsGA2ox2 retained its GA deactivation capability (Fig. 4a) but decreased its overall expression levels was very similar to regulatory hypofunctionalization (Duarte et al. 2006). Therefore, OsGA2ox2 could be recognized as a regulatory hypofunctionalized gene and a minor paralog in the class II OsGA2oxs.

From an evolutionary point of view, the expression difference that results from differences in regulatory motifs between duplicated genes is thought to be a critical way to preserve duplicated genes ( $\mathrm{Li}$ et al. 2005; Ganko et al. 2007). The preservation of the duplicated gene OsGA2ox2 in the rice genome might 

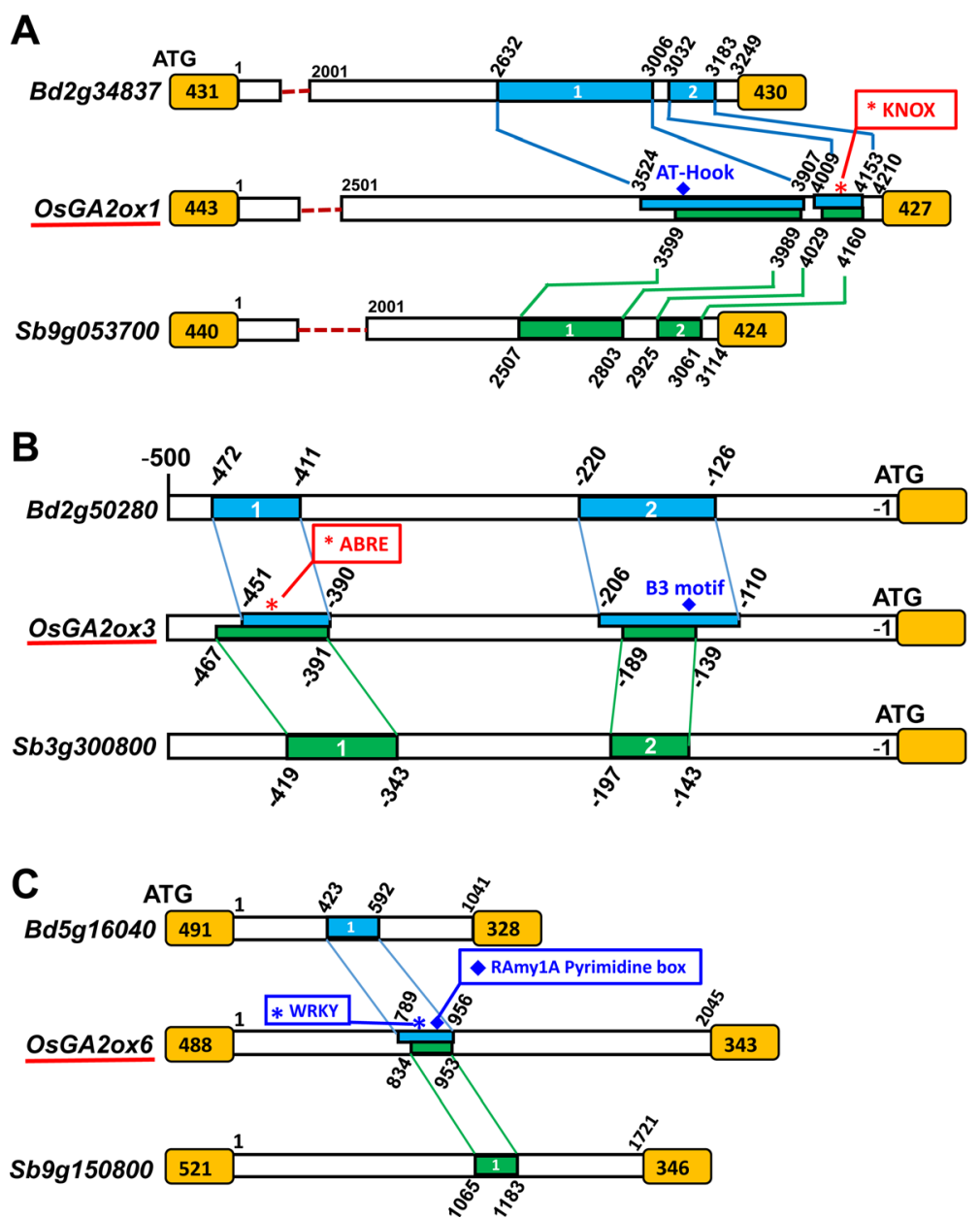

Fig. 10 Conserved sequence motifs identified in the promoter and the first intron regions of rice GA20x1, GA20x3, GA20x6 and their orthologs in B. distachyon and S. bicolor. The sequences $1000 \mathrm{bp}$ upstream of the start codon and the sequences of the first intron of all rice OsGA20xs and their orthologs from B. distachyon and S. bicolor were analyzed by phylogenetic footprinting (http://plantpan.itps.ncku.edu.tw/index.html). The identified conserved regions are colored blue for $B d$ and green for $S b$, and their relative sequence locations in each conserved region are shown. The exons are colored orange, and their sequence lengths are shown. The representative binding motifs with red boxes have been characterized, and those with blue boxes have not yet been characterized. a The relative locations of the AT-Hook motif in region 1 and the KNOX motif in region 2 of the first intron in OSGA2OX1 are indicated. $\mathbf{b}$ The relative locations of the ABRE motif in region 1 and the B3 motif in region 2 of the OsGA20x3 promoter region are indicated. c The relative locations of the WRKY and RAmy1A Pyrmidine box motifs in the first intron of OsGA20x6 are indicated

thus function as a genetic buffer against the detrimental effects caused by deleterious mutations (Duarte et al. 2006; Zhang 2012) or environmental stresses, as evidence has shown that, among 2OGDencoding genes, GA2ox genes are particularly responsive to various abiotic stresses (Colebrook et al. 2014). Another evidence in multiple genes family, such as the three GID1a, GID1b1, and GID1b2 tomato GA receptors, showed that any single- and double-mutant plants were normal under optimal growth conditions, but a high degree of phenotypic variability was observed under changing environments, suggesting that all three genes are needed to maintain phenotypic stability under environmental stress (Illouz-Eliaz et al. 2019). We therefore could not rule out the possibility that the expression of OsGA2ox2 might be induced under certain stress conditions to maintain phenotypic stability, as was the case for multiple GID1 genes. However, future work is needed to explore this possibility.

\section{OsGA2ox4 and OsGA2ox8 can be Recognized as} Regulatory Hypofunctionalized Genes in Clade A of Class I and are Functionally Preserved in the Rice Genome Phylogenetic analysis of the rice GA2ox genes grouped OsGA2ox3, 4 and 8 into clade A of class I (Fig. 5a; Additional file: Fig. S1), and they are located in the syntenic blocks between chromosomes \#1 and \#5 (Additional file: 
Fig. S6b; OsGA2ox1/OsGA2ox2 and OsGA2ox7/ OsGA2ox10 located in their corresponding syntenic blocks on chromosomes \#1 and \#5 were also observed), which was proposed to have occurred through a wholegenome duplication (WGD) event occurring approximately 96 million years ago before the Poaceae species divergence (Wang et al. 2015). Unlike the case of OsGA2ox1 and OsGA2ox2, which have similar exonintron gene structures, the second intron of OsGA2ox4 and the first intron of OsGA2ox8 were lost (Fig. 9a). This intron loss could be explained by either the RNA-based gene conversion model, the alternative-splicing-based model (Zhang et al. 2014) or other complex evolutionary mechanisms that were imposed on these two duplicated genes.

Although the exon-intron gene structures of OsGA2ox4 and OsGA2ox8 differ from the structure of OsGA2ox3, their proteins comprise similar amino acid sequences; moreover, when their full-length cDNAs were overexpressed in transgenic rice, the plants showed the same severe dwarf phenotype as that caused by OsGA2ox3 (Figs. 3d, f; 4a). Even though the same enzyme activity assay performed for OsGA2ox3 (Sakai et al. 2003) and OsGA2ox4 (Liu et al. 2018) was not performed for OsGA2ox8, the GA deactivation capability reflected by plant height reduction was essentially the same among them (Fig. 4a). However, the expression profiles of OsGA2ox4 and OsGA2ox8 were very different from the profile of OsGA2ox3 (Additional file: Fig. S7a, b). Ubiquitous expression of OsGA2ox3 in various tissues has been reported (Sakai et al. 2003) and was further confirmed by at least two rice expression profile databases (Additional file: Fig. S7). By contrast, the expression of OsGA2ox4 and OsGA2ox8 was barely detectable in any tissue (Additional file: Fig. S7).

As mentioned above, expression differences between duplicated genes might be caused by sequence variations in the cis-regulatory motifs between duplicated genes ( $\mathrm{Li}$ et al. 2005). Indeed, number of the sequence variations, counted by the numbers of SNPs/INDELs in the $5^{\prime}$ regulatory regions of OsGA2ox4 and OsGA2ox8, was greater than the number in OsGA2ox3 (Table 2). Additionally, phylogenetic footprinting analysis revealed two conserved regions in the $5^{\prime}$-regulatory region of OsGA2ox3 and its orthologs from B. distachyon and $S$. bicolor (Fig. 10b), but these regions were absent in OsGA2ox4 and OsGA2ox8. These conserved regions that contained several important regulatory motifs (Additional file: Table S2b) might be responsible for the expression differences among OsGA2ox3, 4 and 8 . While the expression of OsGA2ox4 and OsGA2ox8 was barely detectable in any tissue (Additional file: Fig. S7), the expression of OsGA2ox4 in seedlings increased in response to irradiance to reduce the GA content (Hirose et al.
2012), and the expression of OsGA20x8 was reported to be regulated by the miR156-IPA1 regulatory mechanism in controlling seed dormancy (Miao et al. 2019). Overall, we proposed that these two duplicated genes could have evolved via regulatory hypofunctionalization and might function together as a genetic buffer against detrimental mutations, as mentioned above (Duarte et al. 2006; Zhang 2012).

OsGA2ox7 can be Recognized as a Functional Attenuated Gene and can be Used to Create Semidwarf Plants

Phylogenetic analysis in the Poaceae resulted in the division of class I GA2ox genes into clades A and B, and transgenic study demonstrated that the GA deactivation capability was very different between clade A and B genes (Fig. 5b, c). The OsGA2ox7 clade B rice gene, in addition to its low expression levels in most tissues (Additional file: Fig. S7), showed much less GA deactivation capability than its paralogs OsGA2ox3, OsGA2ox4, OsGA2ox8; which draws interest to its functional role in rice and potential applications. It has been reported that the expression of OsGA2ox7 was upregulated in rice seedlings in response to irradiance and was thought to be involved in seedling leaf sheath suppression under light (Hirose et al. 2012). However, the activation of OsGA2ox7 in the T-DNA activation-tagged mutant M66925 showed a very minor effect on plant growth (Fig. 1d), suggesting that the majority of the bioactive GAs that decreased in rice seedlings under irradiance might not be due to elevated OsGA2ox7 expression but instead is due to other elevated expression of other GA2oxs, such as OsGA2ox4, OsGA2ox5, and OsGA2ox6 (Hirose et al. 2012). From an evolutionary point of view, we therefore propose that $O s G A 2 o x 7$ has hypofunctionally evolved in terms of both its transcription and GA deactivation capability and thus might have only a minor role in rice growth and development.

Even though OsGA2ox7 might not be crucially involved in rice growth and development, it may be a good candidate gene for overexpression and genetic engineering and thus improving the plant architecture, stress tolerance and possibly grain yield of rice, similar to the findings in our previous report involving point-mutated OsGA2ox6 variants (Lo et al. 2017). The semidwarfing trait causing approximately $55-60 \% \mathrm{RPH}$ in nontransgenic (NT/WT) plants and an approximately 30\% increase in tiller/panicle numbers (Table 1) makes OsGA2ox7-OX transgenic lines another useful option for future applications.

\section{GA Deactivation Capability Attenuated in Clade B GA2oxs is Associated with Four Amino Acid Variants}

Two functionally distinct effects on growth suppression between clades A and B within class I were first 
observed and demonstrated in the present study (Figs. 4a; $5 \mathrm{~b}, \mathrm{c})$. Thus, we proposed that certain amino acid variations or structural differences between these two clades exist to differentiate their GA deactivation capability. Indeed, the present study demonstrated at least four amino acid variants that are distinct between clade A (C186, C194, Q220 and Y274 in OsGA2ox3) and clade B (R191, P201, E227 and F283 in OsGA2ox7); these variants are associated with strong clade A (such as OsGA2ox3) and weak clade B (such as OsGA2ox7) GA deactivation capability.

Among these four amino acid variants, the functional importance of C186 and C194 in OsGA2ox3 was proposed via $\mathrm{X}$-ray structural analysis and elucidated via an in vitro activity assay (Takehara et al. 2020). The crystal structure showed that the amino acid C194 was responsible for forming intermolecular disulfide bridges (C194-C194) and was crucial for tetramer formation, the $\mathrm{Km}$ for $\mathrm{GA}_{4}$ was reported as being approximately 10 -fold lower for the tetramer with the monomer, while the Vmax was 4-fold higher for the tetramer (Takehara et al. 2020). Further sequence alignment showed that the amino acid C194 was conserved in clade A of class I (OsGA2ox3, 4 and 8) but diverged in clade B (OsGA2ox7 and 10) of class I and class II (OsGA2ox1 and 2) and was absent in class III (OsGA2ox5, 6 and 9). Another conserved amino acid, C186, in clade A but that varied in clade B was described as one of the three amino acids that form the hinge site of the entrance gate for substrate $\mathrm{GA}_{4} \mathrm{~s}$ (Takehara et al. 2020), which might also be responsible for some enzymatic activity differences between clades A and B.

In the present study, overexpressing the OsGA2ox3 C194P variant (replacing C194 with Pro) in transgenic plants $(3 P-O X)$ resulted in minor effects on attenuating GA deactivation capability, increasing the RPH of NT from $13 \%$ to approximately $17 \%$ (Fig. 8c). By contrast, overexpressing the OsGA2ox3 C186R variant (replacing $\mathrm{C} 186$ with Arg) in transgenic plants $(3 R-O X)$ caused noticeable effects on attenuating the GA deactivation capability, increasing the RPH of NT from $13 \%$ to approximately 29\% (Fig. 8c). Enhanced additive effects on attenuating GA deactivation capability that increased the RPH up to approximately $40 \%$ that of NT (Fig. 8c) were observed in transgenic plants $(3 R P-O X)$, in which overexpression of the C186R/C194P double variant (replacing C186 with Arg and C194 with Pro) demonstrated that both $\mathrm{C} 186$ and $\mathrm{C} 194$ were important for OsGA2ox3 activity in planta.

Two other amino acid variations between clades A (Q220 and Y274) and B (E227 and F283) did not involve the key amino acids at the catalytic pocket and did not display any possible interaction with GAs or cofactors in a previous report (Takehara et al. 2020), and $3 E-O X$ and
$3 F-O X$ transgenic plants overexpressing their singleamino acid-replaced variants, showed no obvious effects on GA deactivation capability (Fig. 7). However, 3EF-OX transgenic plants overexpressing the Q220E/Y274F double variant (replacing Q220 with Glu and Y274 with Phe) showed an obvious increase in $\mathrm{RPH}$ - up to approximately $24 \%$ that of NT (Fig. 7a), suggesting that amino acid sequences not involved in the formation of the catalytic pocket of OsGA2ox3 also affected its GA deactivation capability in planta and that a synergistic effect on these two conserved amino acids occurred. Although the Q220 and Y274 residues were outside the catalytic pocket (Additional file: Fig. S3), they were close to the iron (HTD) (Fig. 6b)- and 2OG-binding sites (RVS) (Fig. 6c), which might affect the interaction of OsGA2ox3 with iron and 2OG and thus affect the GA deactivation capability.

Overall, these four amino acids conserved in clade A were associated with their GA deactivation capability, and this capability could be attenuated when the conserved amino acids were replaced with the amino acids conserved in their corresponding site of clade $B$, suggesting that amino acid variations in clade B GA2oxs have evolved to attenuate their GA deactivation capability, such as that which occurred for OsGA2ox7. This evidence supports the different GA deactivation capabilities not only between OsGA2ox3 and OsGA2ox7 but also between clade A (OsGA2ox4 and OsGA2ox8 from rice; $B d 2 g 50280$ and $B d 2 g 19900$ from B. distachyon) and clade B (Bd2g06670, Bd2g32577 from B. distachyon), which was demonstrated in the present study (Figs. 4a; $5)$.

\section{OsGA2ox1, OsGA2ox3 and OsGA2ox6 were Conserved and Dominant in their Respective Classes}

Phylogenetic analysis based on the protein sequences classified GA2oxs into three classes (Lee and Zeevaart 2005), where the rice genes OsGA2ox1 and OsGA2ox2 belong to class II; OsGA2ox3, OsGA2ox4, OsGA2ox7, OsGA2ox8, and OsGA2ox10 belong to class I; and OsGA2ox5, OsGA2ox6, and OsGA2ox9 belong to class III (Additional file: Fig. S1). The cDNAs of nine (OsGA2ox1 to 9) OsGA2ox genes have been demonstrated to be functional and revealed various GA deactivation capabilities based on their effects on plant height reductions (Fig. 4a). The overexpression of OsGA2ox1 in class II, the overexpression of OsGA2ox3, OsGA2ox4, and $O s G A 20 x 8$ in class $\mathrm{I}$ and the overexpression of OsGA2ox6 in class III revealed stronger GA deactivation capability than the others in their respective classes, which raises the question of whether any dominant paralogs exist in each class of the gene family. To explore this question, in addition to comparing their GA deactivation capability, we accounted for the exon-intron 
structures (Fig. 9), sequence differences in the promoter and coding regions (Table 2) and the expression profiles in various tissues (Additional file: Fig. S7) to understand the importance of each OsGA2ox gene in the family.

As the $5^{\prime}$-regulatory region and the intron region close to the transcription start site could be involved in regulating gene expression (Xie et al. 2018) by a mechanism called intron-mediated enhancement (IME) (Laxa 2016), the sequences $1000 \mathrm{bp}$ upstream from the start codon and the sequences of the first intron of all rice OsGA2oxs and their orthologs from B. distachyon and $S$. bicolor were analyzed by phylogenetic footprinting (Chow et al. 2019). This analysis resulted in the identification of three genes, OsGA2ox1, OsGA2ox3 and OsGA2ox6, containing conserved regions: two conserved regions (approximately 306-395 bp of region \#1 and 140-157 bp of region \#2) in the first intron of OsGA2ox1 (Fig. 10a), two conserved regions ( $62-78$ bp of region $\# 1$ and $~ 55-105$ bp of region \#2) in the promoter region of OsGA2ox3 (Fig. 10b) and one conserved region ( $120-170 \mathrm{bp}$ ) in the first intron of OsGA2ox6 (Fig. 10c). The locations and lengths of these conserved regions (Additional file: Table S1) and their detailed binding motifs for transcription factors (TFs/motifs, Additional file: Table S2) are provided. Several conserved motifs, such as the KNOX (Bolduc and Hake 2009), AT-Hook (Matsushita et al. 2007) and GATA motifs (Richter et al. 2013) in the first intron of OsGA2ox1 (Additional file: Table S2a), the ABRE/CE1 motifs (Cantoro et al. 2013) in OsGA2ox3 (Additional file: Table S2b) and the NAC/ NAM (Chen et al. 2015) and WRKY/Pyr motifs (Zhang et al. 2004) in OsGA2ox6 (Additional file: Table S2c), which are involved in GA-mediated growth and development regulations, were conserved across species within their respective classes of GA2ox genes.

For the OsGA2ox1 and OsGA2ox2 genes of class II, although their exon/cDNA sequences were similar, OsGA2ox1 had less sequence variation in both the 5 '-regulatory and coding regions than did OsGA2ox2 (Table 2), which might explain why OsGA2ox1 has a slightly better GA deactivation capability than OsGA2ox2 does (Fig. 4a). In addition, the first intron of OsGA2ox1 contains conserved regulatory motifs that were missing in the intron of OsGA2ox2 (Figs. 9a; 10a), which might reduce OsGA2ox2 RNA expression but maintain the expression of OsGA2ox1 at a significant level in reproductive tissues (Sakamoto et al. 2001; Sakamoto et al. 2004) and at relatively high levels in other tissues (Additional file: Fig. S7). Moreover, a taller and approximately 5-day delay in heading date but with normal anthers and seed setting rate for the osga2ox1 knockout mutant was observed in our preliminary results (Additional file: Fig. S8a), however no phenotypic difference was observed in the osga2ox2 knockout mutant (data not shown). Overall, OsGA2ox1 had stronger selection constraints, maintained higher RNA expression levels, and exhibited a better GA deactivation capability; thus, it could be recognized as the dominant paralog in class II.

For the class I genes, there are 5 members (when OsGA20x10 is included) of the family. Although OsGA2ox4 and OsGA2ox8 showed the same strong GA deactivation capability as OsGA2ox3 did (Fig. 4a), their RNA expression levels were much lower than the level of OsGA2ox3 (Additional file: Fig. S7). This differential expression might be explained by the evidence that OsGA2ox3 has the least $5^{\prime}$-regulatory sequence variation among them, and the conserved 5 '-regulatory motifs in OsGA2ox3 were absent in all other members of the family (Fig. 10b). As mentioned above, OsGA2ox7 can be recognized as a functional attenuated gene and OsGA20x10 was confirmed to be a pseudogene in the present study. Moreover, a recent report (Takehara et al. 2020) and our preliminary results using CRISPR/Cas9 to knock out the OsGA2ox3 gene showed a taller phenotype (Additional file: Fig. S8b), while no phenotypic differences were observed among the osga2ox4, osga2ox7, and osga2ox8 knockout mutants (data not shown). Overall, we propose that OsGA2ox3 is the dominant paralog in class I.

OsGA2ox5, OsGA2ox6 and OsGA2ox9 are $\mathrm{C}_{20}$-type GA2oxs and were categorized into class III. Unlike OsGA2ox5, whose intronless gene structure (Fig. 9a) might have arose via a retroposition model (Han and Zhu 2011), OsGA2ox6 and OsGA2ox9 are phylogenetically conserved (Additional file: Fig. S1) and located in syntenic blocks on chromosomes \#2 and \#4 (Additional file: Fig. S6c), suggesting that OsGA2ox6 and OsGA2ox9 are duplicated genes that evolved through ancient WGD events (Han and Zhu 2011; Wang et al. 2015) before the Poaceae species divergence (Han and Zhu 2011; Wang et al. 2015). These three genes have been functionally characterized (Lo et al. 2008), and OsGA2ox6 displayed higher GA deactivation capability than OsGA2ox5 and OsGA2ox9 did (Fig. 4a).

Although no conserved region in the $5^{\prime}$-regulatory region was found, the $5^{\prime}$-regulatory sequences of OsGA2ox6 were much more conserved than the paralogous OsGA2ox5 and OsGA2ox9 sequences were (Table 2), suggesting that $O s G A 20 x 6$ was under stronger selective constraints, which might be important to maintain its transcriptional ability and specificity. Through phylogenetic footprinting assays, a conserved region in the first intron of OsGA2ox6 (Fig. 10c) that contains transcriptional regulatory motifs, such as NAC/NAM- and WRKY/Pyr-binding motifs, was identified (Additional file: Table S2c). A NAC transcription factor, OsNAC2, is 
involved in suppressing the expression of GA biosynthesis-related genes and enhancing that of GA deactivation-related genes (Chen et al. 2015), and a WRKY transcription factor, OsWRKY71, functions as a transcriptional repressor to repress the expression of the GA-induced $\alpha$-amylase gene $A m y 32 b$ in aleurone cells (Zhang et al. 2004), suggesting that the conserved NAC/ NAM- and WRKY/Pyr-binding motifs in OsGA2ox6 might be important for OsGA2ox6 transcriptional regulation. In addition, the osga2ox6 mutant created by the CRISPR/Cas9 system presented chalky characteristics of its rice grains (Chen et al. 2019, and this study) and exhibited a taller phenotype in our preliminary study (Additional file: Fig. S8c), but the osga2ox9 knockout mutant (Chen et al. 2019) and our osga2ox5 knockout mutant (data not shown) did not show changes in plant height. Overall, we propose that OsGA2ox6 is the dominant paralog in class III.

In summary, OsGA2ox1 in class II, OsGA2ox3 in class I and OsGA2ox6 in class III had the least sequence variation in both the $5^{\prime}$-regulatory and coding regions (Table 2), contained conserved regulatory motifs in either $5^{\prime}$-regulatory or the first intron regions (Fig. 10), and revealed higher RNA expression levels (Additional file: Fig. S7) and stronger GA deactivation capability (Fig. 4a) than others did in their respective classes; thus, they can be recognized as the dominant paralogs in each of their respective classes, with preserved functional importance.

\section{Conclusions}

In the present study we demonstrated that the rice GA2ox gene family contains nine functional genes and the class I GA2ox genes are divided into two functionally distinct clades in Poacea. Among them, the OsGA2ox7 of clade B is a functional attenuated gene and that led to the identification of four conserved amino acids C186/C194 and Q220/Y274 in clade A which were critically associated with GA deactivation capability. In addition, through sequence divergence, RNA expression profile and GA deactivation capability analyses, we proposed that OsGA2ox1, OsGA2ox3 and OsGA2ox6 are the three predominant paralogs in the family.

\section{Methods}

\section{Plant Materials and Growth Conditions}

The rice cultivar Oryza sativa Tainung 67 was used as the wild-type accession in this study, and the TDNA mutants used in this study were obtained from the Taiwan Rice Insertional Mutants library (http:// trim.sinica.edu.tw/). The seeds of wild-type plants and T-DNA mutants were surface sterilized with $2.5 \%$ sodium hypochlorite $(\mathrm{NaClO})$ and then placed on $\mathrm{MS}$ media (Sigma-Aldrich, St. Louis, MO, USA) in a growth chamber at $28{ }^{\circ} \mathrm{C}$ under a $16 \mathrm{~h} / 8 \mathrm{~h}$ light/dark cycle. Approximately 14-21 days later, the grown seedlings were transferred to a greenhouse or an isolated paddy field.

For the exogenous GA feeding experiment, the seeds from nontransgenic (NT) and OsGA2ox activationtagged mutants or overexpression transgenic rice plants were grown on MS media for 7 days and then transferred to MS media consisting of $10 \mu \mathrm{M} \mathrm{GA}$; the shoot length of the seedlings was measured after 3 days of incubation.

Brachypodium distachyon seeds were incubated at $4{ }^{\circ} \mathrm{C}$ for 5-7 days. Then, the testa of seeds was removed, and seeds were placed on sterile water-soaked paper towels in petri dishes $(90 \times 15 \mathrm{~mm})$, which were covered by tinfoil to avoid irradiance. After 2-3 days of incubation at $22^{\circ} \mathrm{C}$, the germinated seeds were transferred to pots in a growth chamber at $22^{\circ} \mathrm{C}$ under a $20 \mathrm{~h} / 4 \mathrm{~h}$ light/dark cycle.

\section{Plasmid Construction and Rice Transformation}

For construction of GA2ox overexpression vectors, the full-length cDNA of GA2oxs from rice and B. distachyon were amplified with their respective cloning primer sets (Additional file: Table S3) together with Phusion HighFidelity DNA Polymerase (Thermo Fisher Scientific, Waltham, MA, USA), after which the respective fulllength GA2ox cDNA was further digested (by SpeI and $K p n I$ digestion) and inserted downstream of the maize ubiquitin (Ubi) promoter in a pAHC18 vector (Bruce et al. 1989). For generation of point mutants in OsGA2ox3, site-directed mutagenesis was conducted as previously described (Lo et al. 2017), with minor modifications. The Ubi:OsGA2ox3 vector was used as a template and amplified by point-mutation primer sets (Additional file: Table S3) in conjunction with Phusion High-Fidelity DNA Polymerase, and the Ubi:OsGA2ox3 template in the PCR product was removed by $D p n I$ digestion. Then, point mutants of the Ubi:OsGA2ox3 vector were purified by an Illustra GFX PCR DNA and Gel Band Purification Kit (GE Healthcare, Chicago, IL, USA). The respective Ubi:GA2ox overexpression vectors were linearized by HindIII digestion and then inserted into a pCAM1301 binary vector (Hajdukiewicz et al. 1994) to form transformation vectors. For construction of CRISPR/Cas9 expression vectors, the potential sgRNAs that target each of the OsGA2ox genes were designed using E-CRISPR (Heigwer et al. 2014) (http:// www.e-crisp.org/E-CRISP/) and these respective pair of sgRNAs (Additional file: Table S3) were synthesized, annealed and integrated into the pRGEB32 vector (Xie et al. 2015) driven by the OsU3 promoter. The resulting plasmid vectors were further transformed into Agrobacterium tumefaciens strain EHA-105 and used for rice 
transformation as previously described (Hiei et al. 1997). At least 10 transgenic $\mathrm{T}_{0}$ lines for overexpression constructs and 3 to 5 transgenic lines for CRISPR knockout mutants were obtained and analyzed. Ten or more transgenic lines were used to measure their plant heights and at least three lines were used for RNA expression analysis. For phenotypic comparisons, one or three representative plants were shown. For analysis of CRISPR knockout mutants, the target site sequence modifications were first analyzed at $\mathrm{T}_{0}$ generation, then confirmed at the next generation and using at least 12 progenies from $T_{1}$ or the following generations to cross out their CRISPR/Cas9 constructs.

\section{Southern Blotting and T-DNA Insertion Site Identification} For Southern blotting assays, $15 \mu \mathrm{g}$ of genomic DNA extracted with CTAB extraction buffer (Doyle and Doyle 1987) was digested by restriction enzymes and subjected to $1 \%(\mathrm{w} / \mathrm{v})$ agarose gel electrophoresis. The fractionated DNA was then transferred to an Amersham Hybond- $\mathrm{N}^{+}$ membrane (GE Healthcare, Chicago, IL, USA) and hybridized with a $\mathrm{P}^{32}$-labeled GUS DNA probe. $\mathrm{P}^{32}$-labeled GUS DNA probes were prepared by an Amersham Rediprime II DNA labeling system (GE Healthcare, Chicago, IL, USA) according to the manufacturer's instructions.

For T-DNA insertion identification, the inverse PCR method was conducted as previously described, with minor modifications (Kim et al. 2011), where $25 \mu \mathrm{g}$ of genomic DNA was digested by $S a c I$ or EcoRI in a $100 \mu \mathrm{L}$ volume for $8 \mathrm{~h}$. Afterward, the digested DNA fragments were purified by the phenol-chloroform extraction method (Sambrook and Russell 2006) and subjected to $100 \mu \mathrm{L}$ of ligation mixture consisting of 15 units of T4 DNA ligase (Promega, Madison, WI, USA), after which the ligation reaction was incubated at $4{ }^{\circ} \mathrm{C}$ for $48 \mathrm{~h}$. The ligation products were further purified by the phenolchloroform extraction method and quantified to $500 \mathrm{ng} /$ $\mu \mathrm{L}$ for inverse PCR, which was conducted by using the LB2-B/Hpt-R and RB/GUS-R primer sets (Additional file: Table S3).

\section{RNA Extraction and Gene Expression Analysis}

For target OsGA2ox gene expression analysis in the T-DNA mutants, since most OsGA2oxs were not expressed in leaves after the transition to the reproductive stage (Lo et al. 2008), flag leaves from wildtype plants and T-DNA mutants at the ripening stage were collected for RNA extraction to investigate whether targeted OsGA2oxs were activated in T-DNA mutants. The shoots of 15-day-old B. distachyon seedlings were used for RNA extraction for $B d 2 g 50280, B d 2 g 19900, B d 2 g 06670$ and $B d 2 g 32577$ gene cloning.
Total RNA was extracted by using TRIzol reagent (Invitrogen, Carlsbad, CA, USA), and RNase-free DNase I (Thermo Fisher Scientific, Waltham, MA, USA) was used to remove possible DNA contaminants from the RNA samples. Five $\mu \mathrm{g}$ of DNA-free RNA was used in cDNA synthesis by a RevertAid First-Strand cDNA Synthesis Kit (Thermo Fisher Scientific, Waltham, MA, USA) according to the recommended protocol of the manufacturer in a $20 \mu \mathrm{L}$ reaction volume. One $\mu \mathrm{L}$ of synthesized cDNA and 0.6 units of GoTaq DNA polymerase (Promega, Madison, WI, USA) were used for PCR for gene expression analysis by the respective genespecific primer sets (Additional file: Table S3) in a $15 \mu \mathrm{L}$ reaction volume.

The expression sequence data of the respective OsGA2oxs in other rice cultivars were downloaded from UniVIO (Kudo et al. 2013) (http://univio.psc.riken.jp/), the Rice Expression Database (RED) (Xia et al. 2017) (http://expression.ic4r.org/) and RiceXPro (Sato et al. 2013) (https://ricexpro.dna.affrc.go.jp/index.html) and then analyzed, and LOC_OsO1g22910 was used as the representative transcript of OsGA2ox2 in these expression data.

\section{Phylogenetic Analysis and SNP Calculation}

In the sequence similarity search, the protein sequence of OsGA2ox7 was used as a query to identify homologs in basal angiosperms (Amborella trichopoda), basal monocots (S. polyrhiza, Z. marina) and Poaceae (B. distachyon, O. sativa, S. bicolor, S. italica) by the BLASTP program, with an E-value threshold of $10^{-5}$; the protein sequences of the investigated species were downloaded from the Phytozome v12 database (https://phytozome. jgi.doe.gov/pz/portal.html\#). The 2OGD protein conserved domain analysis was determined by using Pfam (El-Gebali et al. 2019), as typical GA2oxes have two Pfam domains: DIOX_N (PF14226) and 2OG-FeII_Oxy (PF03171). Protein sequences from the start of DIOX_N to the end of 2OG-FeII_Oxy were aligned by MAFFT version 7 (Katoh and Standley 2013) with the L-INS-I model, and the resulting multiple sequence alignment data were trimmed by trimAl v1.2, with a cutoff value of 0.6 (all columns were removed when the gap percentage surpassed 40\%). Then, the sequences were removed from the alignment if more than $40 \%$ gaps were present. The trimmed alignment data were used in phylogenetic tree construction by RAxML version 8 (Stamatakis 2014) with the parameters -m PROTGAMMAJTT and a bootstrap analysis with 400 replicates.

The sequence variation of the 5 '-regulatory region (1 $\mathrm{kb}$ upstream of the start codon) and coding region among the respective OsGA2oxs were retrieved from Rice Variation Map (RiceVarMap) version 2.0 (Zhao et al. 2015). The number of SNPs/INDELs in the $5^{\prime}$ - 
regulatory region was a combination of both singlenucleotide polymorphisms (SNPs) and insertions/deletions (INDELs) within the region, and the numbers of nonsynonymous $(\mathrm{dN})$, synonymous $(\mathrm{dS})$ or frameshift mutations in the coding region were calculated based on the information within RiceVarMap. For phylogenetic footprinting analysis, the sequences of the $5^{\prime}$-regulatory region ( $1 \mathrm{~kb}$ upstream of the start codon) and intron region of the respective GA2oxs in O. sativa, B. distachyon and S. bicolor were aligned by the "cross species" function of Plant Promoter Analysis Navigator (PlantPan) version 3.0 (http://plantpan.itps.ncku.edu.tw/index.html) (Chow et al. 2019), with an E-value threshold of $10^{-5}$. The exon-intron gene structure of the OsGA2oxs was displayed by Gene Structure Display Server (GSDS) version 2.0 (Hu et al. 2015). The characteristic motifs among class I GA2oxs were identified by Multiple Em for Motif Elicitation (MEME) software, and the parameters were set as previously described (Bailey et al. 2015; Huang et al. 2015).

\section{Statistical Analysis}

All data were analyzed with Statistical Product and Service Solutions (International Business Machines Corporation, Armonk, New York, USA). For multiple comparisons, the Turkey's honestly significant difference (HSD) test was used. Different letters represent differences at a significance level of $P$-value $<0.05$. For comparison with the control, the Student's $t$-test was used, and significance levels are indicated as: * $=$ $0.01<P<0.05,{ }^{* * *}=0.001<P<0.01,{ }^{* * * *}=P<0.001$. The comparisons between the gene-edited plants and wild-type (WT) were presented by means of boxplots.

\section{Accession Numbers and Gene Loci}

The following gene loci can be found in the Phytozome database or RAP-DB:

\begin{tabular}{l}
\hline OsActin: LOC_Os03g61970 \\
OsGA2ox2: Os01g0332200 (RAP-DB) \\
OsGA2ox4: LOC_Os05g43880 \\
OsGA2ox6: LOC_Os04g44150 \\
OsGA2ox8: LOC_Os05g48700 \\
OsGA2ox10: LOC_Os05g11810 \\
OsGA3ox2: LOC_Os01g08220 \\
Bd2g19900: Bradi2g19900 \\
Bd2g16727: Bradi2g16727 \\
Bd2g32577: Bradi2g32577 \\
Sb9g196300: Sobic.009G196300 \\
Sb9g230800: Sobic.009G230800 \\
Si5g323400: Seita.5G323400
\end{tabular}

OsGA20x1: LOC_Os05g06670

OsGA20x3: LOC_Os01g55240

OsGA2ox5: LOC_Os07g01340

OsGA2ox7: LOC_Os01g11150

OsGA20x9: LOC_Os02g41954

OsGA20ox2: LOC_Os01g66100

Bd2g50280: Bradi2g50280

Bd2g06670: Bradi2g06670

Bd2g16750: Bradi2g16750

Sb3g300800: Sobic.003G300800

Sb3g022700: Sobic.003G022700

Sb9g077500: Sobic009G077500

Si5g147400: Seita.5G147400
Accession Numbers and Gene Loci (Continued)

Si3g148100: Seita.3G148100

Si5g266500: Seita.5G266500

Si3g074700: Seita.3G074700

\section{Supplementary Information}

The online version contains supplementary material available at https://doi. org/10.1186/s12284-021-00499-4.

\begin{abstract}
Additional file 1 : Figure S1. Phylogenetic tree based on comparisons of OsGA20x amino acid sequences. Figure S2. Effects of exogenous GA treatment on seedling growth. Figure $\mathbf{S 3}$. Relative positions of amino acids Q220 and Y274 in OsGA20x3 according to the resolved 3-D protein structure. Figure S4. Phenotypic comparison of WT-OX, 3E-OX, 3F-OX and $3 E F-O X$ transgenic rice plants and their RNA expression analysis. Figure S5. Phenotypic comparison of WT-OX, 3R-OX, 3P-OX and 3RP-OX transgenic rice plants and their RNA expression analysis. Figure S6. Locations of and evolutionary relationships among the OsGA20x genes on rice chromosomes. Figure S7. Expression analysis of OsGA20x genes in various tissues according to data collected from different rice expression databases. Figure S8. Phenotypic comparisons of CRISPR/Cas9 knockout OsGA20x1, OsGA20x3, and OsGA20x6 mutants. Table S1A. The locations and aligned lengths of the two conserved regions in the first intron of OsGA20X1 and its orthologs in Bd and Sb. Table S1B. The locations and aligned lengths of the conserved regions in the $5^{\prime}$-regulatory region of OsGA20x3 and its orthologs in Bd and Sb. Table S1C. The locations and aligned lengths of the conserved region in the first intron of OsGA20x6 and its orthologs in Bd and Sb. Table S2A. DNA-binding motifs in the first intron region of OsGA2ox1. Table S2B. DNA-binding motifs in promoter regions of OsGA2ox3. Table S2C. DNA-binding motifs in the first intron region of OsGA20x6. Table S3. List of primers and their sequences used in this study.
\end{abstract}

\section{Acknowledgments}

We thank the Division of Biotechnology, TARI, for providing the green house and isolated field to grow the transgenic rice plants.

\section{Authors' Contributions}

$\mathrm{KTH}$ and LJC consolidated the results, wrote the manuscript and finalized the manuscript. KTH, YTC, TJH, SML, CHH, SYS and SFL performed experiments including genes cloning, vectors construction, T-DNA mutants screening and amino acids variants creation and identification. KTH performed bioinformatics analysis. SHL performed rice transformation and characterized transgenic rice plants at green house; IWW and CST grew and characterized transgenic plants at the isolated field of Taiwan Agriculture Research Institute (TARI). The author(s) read and approved the final manuscript.

\section{Funding}

This work was financially supported by grants (MOST104-2313-B-005-003MY3 and MOST107-2313-B-005-016-MY3 to Liang-Jwu Chen) from Ministry of Science and Technology, Taiwan, and in part by the Advanced Plant Biotechnology Center via the Featured Areas Research Center Program within the framework of the Higher Education Sprout Project by the Ministry of Education (MOE), Taiwan.

Availability of Data and Materials

Not applicable.

\section{Declarations}

Ethics Approval and Consent to Participate

Not applicable.

\section{Consent for Publication}

Not applicable.

Competing Interests

The authors declare that they have no competing interests. 


\section{Author details}

${ }^{1}$ Institute of Molecular Biology, National Chung Hsing University, Taichung 40227, Taiwan. ${ }^{2}$ Biotechnology Center, National Chung Hsing University, Taichung 40227, Taiwan. ${ }^{3}$ Division of Biotechnology, Taiwan Agriculture Research Institute, Taichung 41362, Taiwan.

Received: 1 March 2021 Accepted: 3 June 2021

Published online: 28 July 2021

\section{References}

Bailey TL, Johnson J, Grant CE, Noble WS (2015) The MEME suite. Nucleic Acids Res 43(W1):W39-W49. https://doi.org/10.1093/nar/gkv416

Balakirev ES, Ayala FJ (2003) Pseudogenes: are they "junk" or functional DNA? Annu Rev Genet 37(1):123-151. https://doi.org/10.1146/annurev.genet.37.04 0103.103949

Bolduc N, Hake S (2009) The maize transcription factor KNOTTED1 directly regulates the gibberellin catabolism gene ga20x1. Plant Cell 21(6):1647-1658. https://doi.org/10.1105/tpc.109.068221

Bruce WB, Christensen AH, Klein T, Fromm M, Quail PH (1989) Photoregulation of a phytochrome gene promoter from oat transferred into rice by particle bombardment. Proc Natl Acad Sci U S A 86(24):9692-9696. https://doi.org/1 0.1073/pnas.86.24.9692

Cantoro R, Crocco CD, Benech-Arnold RL, Rodriguez MV (2013) In vitro binding of Sorghum bicolor transcription factors $\mathrm{ABI} 4$ and $\mathrm{ABI}$ to a conserved region of a GA 2-OXIDASE promoter: possible role of this interaction in the expression of seed dormancy. J Exp Bot 64(18):5721-5735. https://doi.org/10.1093/jxb/ ert347

Chen S, Wang X, Zhang L, Lin S, Liu D, Wang Q, Cai S, El-Tanbouly R, Gan L, Wu H, Li Y (2016) Identification and characterization of tomato gibberellin 2oxidases (GA2oxs) and effects of fruit-specific SIGA2ox1 overexpression on fruit and seed growth and development. Hortic Res 3(1):16059. https://doi. org/10.1038/hortres.2016.59

Chen X, Lu S, Wang Y, Zhang X, Lv B, Luo L, Xi D, Shen J, Ma H, Ming F (2015) OsNAC2 encoding a NAC transcription factor that affects plant height through mediating the gibberellic acid pathway in rice. Plant J 82(2):302314. https://doi.org/10.1111/tpj.12819

Chen X, Tian X, Xue L, Zhang X, Yang S, Traw MB, Huang J (2019) CRISPRbased assessment of gene specialization in the gibberellin metabolic pathway in rice. Plant Physiol 180(4):2091-2105. https://doi.org/10.1104/ pp.19.00328

Chow CN, Lee TY, Hung YC, Li GZ, Tseng KC, Liu YH, Kuo PL, Zheng HQ, Chang WC (2019) PlantPAN3.0: a new and updated resource for reconstructing transcriptional regulatory networks from ChIP-seq experiments in plants. Nucleic Acids Res 47(D1):D1155-D1163. https://doi.org/10.1093/nar/gky1081

Colebrook EH, Thomas SG, Phillips AL, Hedden P (2014) The role of gibberellin signalling in plant responses to abiotic stress. J Exp Biol 217(1):67-75. https:// doi.org/10.1242/jeb.089938

Doyle JJ, Doyle JL (1987) A rapid DNA isolation procedure for small quantities of fresh leaf tissue. Phytochem Bull 19:11-15

Duarte JM, Cui L, Wall PK, Zhang Q, Zhang X, Leebens-Mack J, Ma H, Altman N, dePamphilis CW (2006) Expression pattern shifts following duplication indicative of subfunctionalization and neofunctionalization in regulatory genes of Arabidopsis. Mol Biol Evol 23(2):469-478. https://doi.org/10.1093/ molbev/msj051

El-Gebali S, Mistry J, Bateman A, Eddy SR, Luciani A, Potter SC, Qureshi M, Richardson L, Salazar GA, Smart A, Sonnhammer ELL, Hirsh L, Paladin L, Piovesan D, Tosatto SCE, Finn RD (2019) The Pfam protein families database in 2019. Nucleic Acids Res 47(D1):D427-D432. https://doi.org/10.1093/nar/ gky995

Ganko EW, Meyers BC, Vision TJ (2007) Divergence in expression between duplicated genes in Arabidopsis. Mol Biol Evol 24(10):2298-2309. https://doi. org/10.1093/molbev/msm158

Giacomelli L, Rota-Stabelli O, Masuero D, Acheampong AK, Moretto M, Caputi L, Vrhovsek U, Moser C (2013) Gibberellin metabolism in Vitis vinifera L. during bloom and fruit-set: functional characterization and evolution of grapevine gibberellin oxidases. J Exp Bot 64(14):4403-4419. https://doi.org/10.1093/jxb/ ert251

Hajdukiewicz P, Svab Z, Maliga P (1994) The small, versatile PPZP family of Agrobacterium binary vectors for plant transformation. Plant Mol Biol 25(6): 989-994. https://doi.org/10.1007/BF00014672
Han F, Zhu B (2011) Evolutionary analysis of three gibberellin oxidase genes in rice, Arabidopsis, and soybean. Gene 473(1):23-35. https://doi.org/10.1016/j. gene.2010.10.010

Heigwer F, Kerr G, Boutros M (2014) E-CRISP: fast CRISPR target site identification. Nat Methods 11(2):122-123. https://doi.org/10.1038/nmeth.2812

Hiei Y, Komari T, Kubo T (1997) Transformation of rice mediated by Agrobacterium tumefaciens. Plant Mol Biol 35(1/2):205-218. https://doi.org/1 0.1023/A:1005847615493

Hirose F, Inagaki N, Hanada A, Yamaguchi S, Kamiya Y, Miyao A, Hirochika H, Takano M (2012) Cryptochrome and phytochrome cooperatively but independently reduce active gibberellin content in rice seedlings under light irradiation. Plant Cell Physiol 53(9):1570-1582. https://doi.org/10.1093/pcp/ pcs097

Hsing YI, Chern CG, Fan MJ, Lu PC, Chen KT, Lo SF, Sun PK, Ho SL, Lee KW, Wang YC, Huang WL, Ko SS, Chen S, Chen JL, Chung Cl, Lin YC, Hour AL, Wang YW, Chang YC, Tsai MW, Lin YS, Chen YC, Yen HM, Li CP, Wey CK, Tseng CS, Lai MH, Huang SC, Chen LJ, Yu SM (2007) A rice gene activation/knockout mutant resource for high throughput functional genomics. Plant Mol Biol 63(3):351-364. https://doi.org/10.1007/s11103-006-9093-z

Hu B, Jin J, Guo AY, Zhang H, Luo J, Gao G (2015) GSDS 2.0: An upgraded gene feature visualization server. Bioinformatics 31(8):1296-1297. https://doi.org/1 0.1093/bioinformatics/btu817

Huang Y, Wang X, Ge S, Rao GY (2015) Divergence and adaptive evolution of the gibberellin oxidase genes in plants. BMC Evol Biol 15(1):207. https://doi.org/1 $0.1186 / s 12862-015-0490-2$

Illouz-Eliaz N, Ramon U, Shohat H, Blum S, Livne S, Mendelson D, Weiss D (2019) Multiple gibberellin receptors contribute to phenotypic stability under changing environments. Plant Cell 31(7):1506-1519. https://doi.org/10.1105/ tpc. 19.00235

Itoh H, Ueguchi-Tanaka M, Sentoku N, Kitano H, Matsuoka M, Kobayashi M (2001) Cloning and functional analysis of two gibberellin 3 beta -hydroxylase genes that are differently expressed during the growth of rice. Proc Natl Acad Sci U S A 98(15):8909-8914. https://doi.org/10.1073/pnas.141239398

Jasinski S, Piazza P, Craft J, Hay A, Woolley L, Rieu I, Phillips A, Hedden P, Tsiantis M (2005) KNOX action in Arabidopsis is mediated by coordinate regulation of cytokinin and gibberellin activities. Curr Biol 15(17):1560-1565. https://doi. org/10.1016/j.cub.2005.07.023

Jupe F, Rivkin AC, Michael TP, Zander M, Motley ST, Sandoval JP, Slotkin RK, Chen H, Castanon R, Nery JR, Ecker JR (2019) The complex architecture and epigenomic impact of plant T-DNA insertions. PLoS Genet 15(1):e1007819. https://doi.org/10.1371/journal.pgen.1007819

Katoh K, Standley DM (2013) MAFFT multiple sequence alignment software version 7: improvements in performance and usability. Mol Biol Evol 30(4): 772-780. https://doi.org/10.1093/molbev/mst010

Kawahara Y, de la Bastide M, Hamilton JP, Kanamori H, McCombie WR, Ouyang S, Schwartz DC, Tanaka T, Wu J, Zhou S, Childs KL, Davidson RM, Lin H, Quesada-Ocampo L, Vaillancourt B, Sakai H, Lee SS, Kim J, Numa H, Itoh T, Buell CR, Matsumoto T (2013) Improvement of the Oryza sativa Nipponbare reference genome using next generation sequence and optical map data. Rice 6(1):4. https://doi.org/10.1186/1939-8433-6-4

Kawai Y, Ono E, Mizutani M (2014) Evolution and diversity of the 2-oxoglutaratedependent dioxygenase superfamily in plants. Plant J 78(2):328-343. https:// doi.org/10.1111/tpj.12479

Kelley LA, Mezulis S, Yates CM, Wass MN, Sternberg MJ (2015) The Phyre2 web portal for protein modeling, prediction and analysis. Nat Protoc 10(6):845858. https://doi.org/10.1038/nprot.2015.053

Kim GB, Son SU, Yu HJ, Mun JH (2019) MtGA20x10 encoding C $20-G A 2-$ oxidase regulates rhizobial infection and nodule development in Medicago truncatula. Sci Rep 9(1):5952. https://doi.org/10.1038/s41598-01 9-42407-3

Kim SR, Jeon JS, An G (2011) Development of an efficient inverse PCR method for isolating gene tags from T-DNA insertional mutants in rice. Methods Mol Biol 678:139-146. https://doi.org/10.1007/978-1-60761-682-5_11

Kudo T, Akiyama K, Kojima M, Makita N, Sakurai T, Sakakibara H (2013) UniVIO: a multiple omics database with hormonome and transcriptome data from rice. Plant Cell Physiol 54(2):e9. https://doi.org/10.1093/pcp/pct003

Kuroha T, Nagai K, Gamuyao R, Wang DR, Furuta T, Nakamori M, Kitaoka T, Adach K, Minami A, Mori Y, Mashiguchi K, Seto Y, Yamaguchi S, Kojima M, Sakakibara H, Wu J, Ebana K, Mitsuda N, Ohme-Takagi M, Yanagisawa S, Yamasaki M, Yokoyama R, Nishitani K, Mochizuki T, Tamiya G, McCouch SR, Ashikari M (2018) Ethylene-gibberellin signaling underlies adaptation of rice 
to periodic flooding. Science 361(6398):181-186. https://doi.org/10.1126/ science.aat1577

Lange T, Lange MJP (2020) The multifunctional dioxygenases of gibberellin synthesis. Plant Cell Physiol 61(11):1869-1879. https://doi.org/10.1093/pcp/ pcaa051

Laxa M (2016) Intron-mediated enhancement: a tool for heterologous gene expression in plants? Front Plant Sci 7:1977

Lee DJ, Zeevaart JA (2005) Molecular cloning of GA 2-oxidase3 from spinach and its ectopic expression in Nicotiana sylvestris. Plant Physiol 138(1):243-254 https://doi.org/10.1104/pp.104.056499

Li C, Zheng L, Wang X, Hu Z, Zheng Y, Chen Q, Hao X, Xiao X, Wang X, Wang G, Zhang $Y$ (2019) Comprehensive expression analysis of Arabidopsis GA2oxidase genes and their functional insights. Plant Sci 285:1-13. https://doi. org/10.1016/j.plantsci.2019.04.023

Li WH, Yang J, Gu X (2005) Expression divergence between duplicate genes. Trends Genet 21(11):602-607. https://doi.org/10.1016/j.tig.2005.08.006

Li Z, Zhang H, Ge S, Gu X, Gao G, Luo J (2009) Expression pattern divergence of duplicated genes in rice. BMC Bioinfo 10(Suppl 6):S8

Liao CC, Chen LJ, Lo SF, Chen CW, Chu YW (2019) EAT-Rice: a predictive model for flanking gene expression of T-DNA insertion activation-tagged rice mutants by machine learning approaches. PLoS Comput Biol 15(5):e1006942. https://doi.org/10.1371/journal.pcbi.1006942

Lieberman-Lazarovich M, Yahav C, Israeli A, Efroni I (2019) Deep conservation of cis-element variants regulating plant hormonal responses. Plant Cell 31(11): 2559-2572. https://doi.org/10.1105/tpc.19.00129

Liu C, Zheng S, Gui J, Fu C, Yu H, Song D, Shen J, Qin P, Liu X, Han B, Yang Y, Li L (2018) Shortened basal internodes encodes a gibberellin 2-oxidase and contributes to lodging resistance in rice. Mol Plant 11(2):288-299. https://doi. org/10.1016/j.molp.2017.12.004

Lo SF, Ho TD, Liu YL, Jiang MJ, Hsieh KT, Chen KT, Yu LC, Lee MH, Chen CY, Huang TP, Kojima M, Sakakibara H, Chen LJ, Yu SM (2017) Ectopic expression of specific GA2 oxidase mutants promotes yield and stress tolerance in rice. Plant Biotechnol J 15(7):850-864. https://doi.org/1 $0.1111 /$ pbi.12681

Lo SF, Yang SY, Chen KT, Hsing Yl, Zeevaart JA, Chen LJ, Yu SM (2008) A novel class of gibberellin 2-oxidases control semidwarfism, tillering, and root development in rice. Plant Cell 20(10):2603-2618. https://doi.org/10.1105/ tpc.108.060913

MacMillan J (2001) Occurrence of gibberellins in vascular plants, fungi, and bacteria. J Plant Growth Regul 20(4):387-442. https://doi.org/10.1007/s00344 0010038

Magome H, Yamaguchi S, Hanada A, Kamiya Y, Oda K (2008) The DDF1 transcriptional activator upregulates expression of a gibberellin-deactivating gene, GA20x7, under high-salinity stress in Arabidopsis. Plant J 56(4):613-626. https://doi.org/10.1111/j.1365-313X.2008.03627.x

Matsushita A, Furumoto T, Ishida S, Takahashi Y (2007) AGF1, an AT-hook protein, is necessary for the negative feedback of AtGA3ox1 encoding GA 3-oxidase. Plant Physiol 143(3):1152-1162. https://doi.org/10.1104/pp.106.093542

Miao C, Wang Z, Zhang L, Yao J, Hua K, Liu X, Shi H, Zhu JK (2019) The grain yield modulator miR156 regulates seed dormancy through the gibberellin pathway in rice. Nat Commun 10(1):3822. https://doi.org/10.1038/s41467-01 9-11830-5

Moghe GD, Last RL (2015) Something old, something new: conserved enzymes and the evolution of novelty in plant specialized metabolism. Plant Physiol 169:1512-1523

Panchy N, Lehti-Shiu M, Shiu SH (2016) Evolution of gene duplication in plants. Plant Physiol 171(4):2294-2316. https://doi.org/10.1104/pp.16.00523

Pearce S, Huttly AK, Prosser IM, Li YD, Vaughan SP, Gallova B, Patil A, Coghill JA, Dubcovsky J, Hedden P, Phillips AL (2015) Heterologous expression and transcript analysis of gibberellin biosynthetic genes of grasses reveals novel functionality in the GA3ox family. BMC Plant Biol 15(1):130. https://doi.org/1 0.1186/s12870-015-0520-7

Qian W, Liao BY, Chang AY, Zhang J (2010) Maintenance of duplicate genes and their functional redundancy by reduced expression. Trends Genet 26(10): 425-430. https://doi.org/10.1016/j.tig.2010.07.002

Renny-Byfield S, Gallagher JP, Grover CE, Szadkowski E, Page JT, Udall JA, Wang X, Paterson AH, Wendel JF (2014) Ancient gene duplicates in Gossypium (cotton) exhibit near-complete expression divergence. Genome Biol Evol 6(3): 559-571. https://doi.org/10.1093/gbe/evu037

Richter R, Bastakis E, Schwechheimer C (2013) Cross-repressive interactions between SOC1 and the GATAs GNC and GNL/CGA1 in the control of greening, cold tolerance, and flowering time in Arabidopsis. Plant Physiol 162(4):1992-2004. https://doi.org/10.1104/pp.113.219238

Rieu I, Eriksson S, Powers SJ, Gong F, Griffiths J, Woolley L, Benlloch R, Nilsson O, Thomas SG, Hedden P, Phillips AL (2008) Genetic analysis reveals that $C_{19}-G A$ 2-oxidation is a major gibberellin inactivation pathway in Arabidopsis. Plant Cell 20(9):2420-2436. https://doi.org/10.1105/tpc.108.058818

Rodriguez MV, Mendiondo GM, Cantoro R, Auge GA, Luna V, Masciarelli O, Benech-Arnold RL (2012) Expression of seed dormancy in grain sorghum lines with contrasting pre-harvest sprouting behavior involves differential regulation of gibberellin metabolism genes. Plant Cell Physiol 53(1):64-80. https://doi.org/10.1093/pcp/pcr154

Rutter MT, Wieckowski YM, Murren CJ, Strand AE (2017) Fitness effects of mutation: testing genetic redundancy in Arabidopsis thaliana. J Evol Biol 30(6):1124-1135. https://doi.org/10.1111/jeb.13081

Sakai H, Lee SS, Tanaka T, Numa H, Kim J, Kawahara Y, Wakimoto H, Yang CC, Iwamoto M, Abe T, Yamada Y, Muto A, Inokuchi H, Ikemura T, Matsumoto T, Sasaki T, Itoh T (2013) Rice annotation project database (RAP-DB): an integrative and interactive database for rice genomics. Plant Cell Physiol 54(2):e6. https://doi.org/10.1093/pcp/pcs183

Sakai M, Sakamoto T, Saito T, Matsuoka M, Tanaka H, Kobayashi M (2003) Expression of novel rice gibberellin 2-oxidase gene is under homeostatic regulation by biologically active gibberellins. J Plant Res 116(2):161-164. https://doi.org/10.1007/s10265-003-0080-z

Sakamoto T, Kobayashi M, Itoh H, Tagiri A, Kayano T, Tanaka H, Iwahori S, Matsuoka M (2001) Expression of a gibberellin 2-oxidase gene around the shoot apex is related to phase transition in rice. Plant Physiol 125(3):15081516. https://doi.org/10.1104/pp.125.3.1508

Sakamoto T, Miura K, Itoh H, Tatsumi T, Ueguchi-Tanaka M, Ishiyama K, Kobayashi M, Agrawal GK, Takeda S, Abe K, Miyao A, Hirochika H, Kitano H, Ashikari M, Matsuoka M (2004) An overview of gibberellin metabolism enzyme genes and their related mutants in rice. Plant Physiol 134(4):1642-1653. https://doi. org/10.1104/pp.103.033696

Sambrook J, Russell DW (2006) Purification of nucleic acids by extraction with phenol:chloroform. CSH Protoc 2006(1):pdb.prot4455. https://doi.org/10.1101/ pdb.prot4455

Sato Y, Takehisa H, Kamatsuki K, Minami H, Namiki N, Ikawa H, Ohyanagi H, Sugimoto K, Antonio BA, Nagamura Y (2013) RiceXPro version 3.0: expanding the informatics resource for rice transcriptome. Nucleic Acids Res 41(D1): D1206-D1213. https://doi.org/10.1093/nar/gks1125

Schrodinger, L.L.C. (2015). The PyMOL Molecular Graphics System, Version 1.8 (Schrodinger LLC).

Schomburg FM, Bizzell CM, Lee DJ, Zeevaart JA, Amasino RM (2003) Overexpression of a novel class of gibberellin 2-oxidases decreases gibberellin levels and creates dwarf plants. Plant Cell 15(1):151-163. https:// doi.org/10.1105/tpc.005975

Schrager-Lavelle A, Gath NN, Devisetty UK, Carrera E, Lopez-Diaz I, Blazquez MA, Maloof JN (2019) The role of a class III gibberellin 2-oxidase in tomato internode elongation. Plant J 97(3):603-615. https://doi.org/1 0.1111/tpj.14145

Stamatakis A (2014) RAxML version 8: a tool for phylogenetic analysis and postanalysis of large phylogenies. Bioinformatics 30(9):1312-1313. https:/doi. org/10.1093/bioinformatics/btu033

Takehara S, Sakuraba S, Mikami B, Yoshida H, Yoshimura H, Itoh A, Endo M, Watanabe N, Nagae T, Matsuoka M, Ueguchi-Tanaka M (2020) A common allosteric mechanism regulates homeostatic inactivation of auxin and gibberellin. Nat Commun 11(1):2143. https://doi.org/10.1038/s41467-020-1 6068-0

Thomas SG, Phillips AL, Hedden P (1999) Molecular cloning and functional expression of gibberellin 2- oxidases, multifunctional enzymes involved in gibberellin deactivation. Proc Natl Acad Sci U S A 96(8):4698-4703. https:// doi.org/10.1073/pnas.96.8.4698

Toyomasu T, Kawaide H, Sekimoto H, Von Numers C, Phillips AL, Hedden P, Kamiya Y (1997) Cloning and characterization of a cDNA encoding gibberellin 20-oxidase from rice (Oryza sativa) seedlings. Physiol Plant 99(1): 111-118. https://doi.org/10.1111/j.1399-3054.1997.tb03438.x

Wang H, Caruso LV, Downie AB, Perry SE (2004) The embryo MADS domain protein AGAMOUS-like 15 directly regulates expression of a gene encoding an enzyme involved in gibberellin metabolism. Plant Cell 16(5):1206-1219. https://doi.org/10.1105/tpc.021261

Wang X, Wang J, Jin D, Guo H, Lee TH, Liu T, Paterson AH (2015) Genome alignment spanning major poaceae lineages reveals heterogeneous 
evolutionary rates and alters inferred dates for key evolutionary events. Mol Plant 8(6):885-898. https://doi.org/10.1016/j.molp.2015.04.004

Xia L, Zou D, Sang J, Xu X, Yin H, Li M, Wu S, Hu S, Hao L, Zhang Z (2017) Rice expression database (RED): An integrated RNA-Seq-derived gene expression database for rice. J Genet Genomics 44(5):235-241. https://doi.org/10.1016/j. jgg.2017.05.003

Xie K, Minkenberg B, Yang Y (2015) Boosting CRISPR/Cas9 multiplex editing capability with the endogenous tRNA-processing system. Proc Natl Acad Sci U S A 112(11):3570-3575. https://doi.org/10.1073/pna S. 1420294112

Xie Y, Zhang Y, Han J, Luo J, Li G, Huang J, Wu H, Tian Q, Zhu Q, Chen Y, Kawano Y, Liu YG, Chen L (2018) The Intronic cis element SE1 recruits transacting repressor complexes to repress the expression of ELONGATED UPPERMOST INTERNODE1 in Rice. Mol Plant 11(5):720-735. https://doi.org/1 0.1016/j.molp.2018.03.001

Yamaguchi S (2008) Gibberellin metabolism and its regulation. Annu Rev Plant Biol 59(1):225-251. https://doi.org/10.1146/annurev.arplant.59.032 607.092804

Yang L, Takuno S, Waters ER, Gaut BS (2011) Lowly expressed genes in Arabidopsis thaliana bear the signature of possible pseudogenization by promoter degradation. Mol Biol Evol 28(3):1193-1203. https://doi.org/10.1 093/molbev/msq298

Yoshida H, Takehara S, Mori M, Ordonio RL, Matsuoka M (2020) Evolution of GA metabolic enzymes in land plants. Plant Cell Physiol 61(11):1919-1934. https://doi.org/10.1093/pcp/pcaa126

Zhang C, Gschwend AR, Ouyang Y, Long M (2014) Evolution of gene structural complexity: An alternative-splicing-based model accounts for introncontaining retrogenes. Plant Physiol 165(1):412-423. https://doi.org/10.1104/ pp. 113.231696

Zhang J (2012) Genetic redundancies and their evolutionary maintenance. Adv Exp Med Biol 751:279-300

Zhang ZL, Xie Z, Zou X, Casaretto J, Ho TH, Shen QJ (2004) A rice WRKY gene encodes a transcriptional repressor of the gibberellin signaling pathway in aleurone cells. Plant Physiol 134(4):1500-1513. https://doi.org/10.1104/pp.103. 034967

Zhao H, Yao W, Ouyang Y, Yang W, Wang G, Lian X, Xing Y, Chen L, Xie W (2015) RiceVarMap: a comprehensive database of rice genomic variations. Nucleic Acids Res 43(D1):D1018-D1022. https://doi.org/10.1 093/nar/gku894

Zou C, Lehti-Shiu MD, Thibaud-Nissen F, Prakash T, Buell CR, Shiu SH (2009) Evolutionary and expression signatures of pseudogenes in Arabidopsis and rice. Plant Physiol 151(1):3-15. https://doi.org/10.1104/ pp.109.140632

\section{Publisher's Note}

Springer Nature remains neutral with regard to jurisdictional claims in published maps and institutional affiliations.

\section{Submit your manuscript to a SpringerOpen ${ }^{\circ}$ journal and benefit from:}

- Convenient online submission

- Rigorous peer review

- Open access: articles freely available online

- High visibility within the field

- Retaining the copyright to your article

Submit your next manuscript at $\boldsymbol{\nabla}$ springeropen.com 